\title{
Meilleures constantes dans le théorème d'inclusion de Sobolev
}

\author{
par \\ Emmanuel HEBEY \\ 9, rue Villehardouin, 75003 Paris, France et Université de Cergy-Pontoise, \\ Dept. de Mathématiques, avenue du Parc, 8 le Campus, \\ 95033 Cergy-Pontoise Cedex, France.
}

et

Michel VAUGON

58, rue de la Mare-Aubry, 02400 Château-Thierry, France

et Université Paris 6, Dept. de Mathématiques,

4, place Jussieu, 75252 Paris Cedex 05, France.

RÉSUMÉ. - En 1976, Aubin énonçait la conjecture suivante : pour toute variété riemannienne compacte de dimension $N \geq 3$, la meilleure constante de l'inclusion de $H_{1}$ dans $L^{2 N /(N-2)}$ est atteinte. On montre que la conjecture est vraie.

ABSTRACT. - In 1976, Aubin stated the following conjecture: for any compact riemannian manifold of dimension $N \geq 3$, the best constant corresponding to the imbedding of $H_{1}$ in $L^{2 N /(N-2)}$ is attained. We prove that the conjecture is true.

\section{INTRODUCTION ET RÉSULTATS}

Soit $(M, g)$ une variété riemannienne $C^{\infty}$ compacte (sans bord) de dimension $N \geq 3$. Une fois pour toutes, on note

$$
p=\frac{N+2}{N-2}
$$




$$
\begin{aligned}
& \omega_{N}=\text { volume de la sphère unité de } \mathbb{R}^{N+1} \\
& S_{N}=\frac{4}{N(N-2) \omega_{N}^{2 / N}} .
\end{aligned}
$$

$H_{1}(M)$ désigne l'espace de Sobolev complété de $C^{\infty}(M)$ pour la norme :

$$
\|u\|^{2}=\int_{M}\left|\nabla_{g} u\right|^{2} d v(g)+\int_{M} u^{2} d v(g) .
$$

$M$ étant compacte, $H_{1}(M)$ ne dépend pas de $g$.

On sait que $H_{1}(M)$ se plonge continûment dans $L^{p+1}(M)$. De plus (voir Aubin [2]) :

(i) Pour tout $\varepsilon>0$, il existe une constante $C_{\varepsilon}>0$ telle que pour tout $u \in H_{1}(M)$,

$$
\left(\int_{M}|u|^{p+1} d v(g)\right)^{2 /(p+1)} \leq\left(S_{N}+\varepsilon\right) \int_{M}\left|\nabla_{g} u\right|^{2} d v(g)+C_{\varepsilon} \int_{M} u^{2} d v(g) .
$$

(ii) Si pour tout $u \in H_{1}(M)$,

$$
\left(\int_{M}|u|^{p+1} d v(g)\right)^{2 /(p+1)} \leq A \int_{M}\left|\nabla_{g} u\right|^{2} d v(g)+C \int_{M} u^{2} d v(g)
$$

où $A$ et $C$ sont des constantes, alors $A \geq S_{N}$.

On démontre ici que la conjecture énoncée par Aubin dans [3] est vraie. Autrement dit :

THÉORÈME. - Soit $(M, g)$ une variété riemannienne compacte de dimension $N \geq 3$. Il existe alors une constante $C>0$ telle que pour tout $u \in H_{1}(M)$,

$$
\left(\int_{M}|u|^{p+1} d v(g)\right)^{2 /(p+1)} \leq S_{N} \int_{M}\left|\nabla_{g} u\right|^{2} d v(g)+C \int_{M} u^{2} d v(g) .
$$

Ce résultat fut démontré dans [3] lorsque $g$ est à courbure sectionnelle constante, puis dans [16] lorsque $g$ est conformément plate. Par ailleurs, le résultat reste valable pour les variétés compactes à bord lorsque $H_{1}$ est remplacé par $H_{0,1}$ (le complété de $\mathcal{D}(M)$ pour la même norme). Pous plus de détails, on renvoie à l'appendice 1 .

Enfin, la démonstration du théorème passant par plusieurs étapes, le plan de l'article est le suivant :

1. Introduction et résultats

2. Réduction du problème à la boule unité $B$ 
3. Les équations associées à la conjecture

4. Théorie classique des points de concentration

5. Estimations de la vitesse de convergence des $x_{\alpha}$ au bord de $B$

6. Transformation du problème et blow-up

7. Une estimée $C^{0}$ pour $\tilde{v}_{\alpha}$

8. Conclusion et identité de Pohozaev

9. Appendices

\section{RÉDUCTION DU PROBLÈME À LA BOULE UNITÉ $B$}

On note $B$ la boule unité de $\mathbb{R}^{N}$. Soit $g$ une métrique riemannienne $C^{\infty}$ définie sur $\bar{B}$ (que l'on pourra voir comme la restriction à $\bar{B}$ d'une métrique riemannienne $C^{\infty}$ définie sur $\mathbb{R}^{N}$ ). On note $H_{0,1}(B)$ l'espace de Sobolev complété de $\mathcal{D}(B)$, l'espace des fonctions $C^{\infty}$ à support compact dans $B$, pour la norme

$$
\|u\|^{2}=\int_{B}\left|\nabla_{g} u\right|^{2} d v(g)+\int_{B} u^{2} d v(g) .
$$

$\bar{B}$ étant compacte, là encore $H_{0,1}(B)$ ne dépend pas de la métrique $g$.

On dira que $g$ vérifie la propriété $(*)$ sur $\bar{B}$ s'il existe une boule ouverte $B^{\prime}$ centrée en 0 , contenant $\bar{B}$, telle que $g$ soit géodésiquement convexe sur $B$ et sur $B^{\prime}$ (i.e. deux points quelconques de $B$, resp. $B^{\prime}$, sont joints par une unique géodésique contenue dans $B$, resp. $B^{\prime}$, et cette géodésique est minimisante).

On démontre ici la proposition suivante.

Proposition 1. - Soit B la boule unité de $\mathbb{R}^{N}, N \geq 3$. On suppose que pour toute métrique $g$ définie sur $\bar{B}$ et vérifiant la propriété $(*)$ sur $\bar{B}$, il existe une constante $C_{g}>0$ telle que pour tout $u \in H_{0,1}(B)$,

$$
\left(\int_{B}|u|^{p+1} d v(g)\right)^{2 /(p+1)} \leq S_{N} \int_{B}\left|\nabla_{g} u\right|^{2} d v(g)+C_{g} \int_{B} u^{2} d v(g) .
$$

La conjecture est alors vérifiée par toute variété riemannienne compacte de dimension $N$. Autrement dit, $(M, g)$ désignant une variété riemannienne compacte quelconque de dimension $N$, il existe une constante $C>0$ telle que pour tout $u \in H_{1}(M)$,

$$
\left(\int_{M}|u|^{p+1} d v(g)\right)^{2 /(p+1)} \leq S_{N} \int_{M}\left|\nabla_{g} u\right|^{2} d v(g)+C \int_{M} u^{2} d v(g) .
$$


Démonstration. - $M$ étant compacte, il existe un atlas fini $\left(U_{i}, \Phi_{i}\right)_{i=1, \ldots, k}$ de $M$, tel que pour tout $i: \Phi_{i}\left(U_{i}\right)=B$

$$
\left(\Phi_{i}^{-1}\right)^{*} g \text { vérifie }(*) \operatorname{sur} \bar{B} \text {. }
$$

Il s'agit là d'un résultat classique de géométrie riemannienne. Pour plus de détails, on renvoie à [17], tome 1 , théorèmes 8.7 page 149 et 3.6 page 166 .

Soit maintenant $\left(\eta_{i}\right)_{i=1, \ldots, k}$ une partition de l'unité subordonnée au recouvrement $\left(U_{i}\right)_{i=1, \ldots, k}$. Sans perdre en généralité, on pourra supposer que pour tout $i$ :

$$
\begin{gathered}
\eta_{i} \text { et } \sqrt{\eta_{i}} \in H_{0,1}\left(U_{i}\right) \cap C^{0}\left(\bar{U}_{i}\right) \\
\left|\nabla_{g} \sqrt{\eta_{i}}\right| \in C^{0}\left(\bar{U}_{i}\right) .
\end{gathered}
$$

Soit maintenant $u \in C^{\infty}(M)$. On pose $u_{i}=\sqrt{\eta_{i}} u$. On a alors :

$$
\left(\int_{M}|u|^{p+1} d v(g)\right)^{2 /(p+1)} \leq \sum_{i=1}^{k}\left(\int_{M}\left|u_{i}\right|^{p+1} d v(g)\right)^{2 /(p+1)} .
$$

Par ailleurs, puisque $u_{i} \in H_{0,1}\left(U_{i}\right)$, les hypothèses de la proposition nous permettent d'écrire qu'il existe une constante $C_{i}>0$ telle que

$$
\left(\int_{M}\left|u_{i}\right|^{p+1} d v(g)\right)^{2 /(p+1)} \leq S_{N} \int_{M}\left|\nabla_{g} u_{i}\right|^{2} d v(g)+C_{i} \int_{M} u_{i}^{2} d v(g) .
$$

On obtient ainsi :

$$
\begin{aligned}
& \left(\int_{M}|u|^{p+1} d v(g)\right)^{2 /(p+1)} \\
& \leq \sum_{i=1}^{k}\left(S_{N} \int_{M}\left|\nabla_{g} u_{i}\right|^{2} d v(g)+C_{i} \int_{M} u_{i}^{2} d v(g)\right) \\
& \leq S_{N} \sum_{i=1}^{k} \int_{M} \eta_{i}\left|\nabla_{g} u\right|^{2} d v(g)+S_{N} \sum_{i=1}^{k} \int_{M} u\left(\nabla_{g} u \nabla_{g} \eta_{i}\right) d v(g) \\
& \quad+S_{N} \sum_{i=1}^{k} \int_{M} u^{2}\left|\nabla_{g} \sqrt{\eta_{i}}\right|^{2} d v(g)+\sum_{i=1}^{k} C_{i} \int_{M} \eta_{i} u^{2} d v(g) \\
& \leq S_{N} \int_{M}\left|\nabla_{g} u\right|^{2} d v(g)+C \int_{M} u^{2} d v(g) \\
& \text { où } C=S_{N} \operatorname{Sup}_{M}\left(\sum_{i=1}^{k}\left|\nabla_{g} \sqrt{\eta_{i}}\right|^{2}\right)+\operatorname{Sup}_{i} C_{i} .
\end{aligned}
$$

On retrouve donc l'inégalité qu'il fallait obtenir. La proposition est démontrée. 


\section{LES ÉQUATIONS ASSOCIÉES À LA CONJECTURE}

En vertu de ce qui vient d'être dit, le problème devient : montrer que pour toute métrique riemannienne $g$ définie sur $\bar{B}$ et vérifiant la propriété (*) sur $\bar{B}$, il existe une constante $C_{g}>0$ telle que pour tout $u \in H_{0,1}(B)$,

$$
\left(\int_{B}|u|^{p+1} d v(g)\right)^{2 /(p+1)} \leq S_{N} \int_{B}\left|\nabla_{g} u\right|^{2} d v(g)+C_{g} \int_{B} u^{2} d v(g)
$$

Soit alors $g$ une métrique riemannienne définie sur $\bar{B}$ et vérifiant la propriété $\left(^{*}\right)$ sur $\bar{B}$. Pour tout réel $\alpha>0$ on définit la fonctionnelle $I_{\alpha}(u)$, $u \in H_{0,1}(B), u \not \equiv 0$, en posant

$$
I_{\alpha}(u)=\frac{\int_{B}\left|\nabla_{g} u\right|^{2} d v(g)+\alpha \int_{B} u^{2} d v(g)}{\left(\int_{B}|u|^{p+1} d v(g)\right)^{2 /(p+1)}} .
$$

Démontrer l'existence de la constante $C_{g}$ revient alors à montrer qu'il existe un réel $\alpha>0$ pour lequel $\operatorname{Inf}_{u} I_{\alpha}(u) \geq \frac{1}{S_{N}}$ (l'inf étant pris sur les $\left.u \in H_{0,1}(B), u \not \equiv 0\right)$.

Par suite, si l'on suppose que $C_{g}$ n'existe pas, on obtient que pour tout $\alpha>0, \operatorname{Inf}_{u} I_{\alpha}(u)<\frac{1}{S_{N}}$. En utilisant des techniques variationnelles standard on démontre alors le résultat suivant.

Proposition 2. - Si pour tout $\alpha>0, \operatorname{Inf}_{u} I_{\alpha}(u)<\frac{1}{S_{N}}$, alors pour tout $\alpha>0$, il existe $\Phi_{\alpha} \in C^{2}(\bar{B}) \cap H_{0,1}(B), \Phi_{\alpha}>0$ sur $B$, et il existe $\left.\lambda_{\alpha} \in\right] 0, \frac{1}{S_{N}}[$, tels que :

$$
\begin{gathered}
\Delta_{g} \Phi_{\alpha}+\alpha \Phi_{\alpha}=\lambda_{\alpha} \Phi_{\alpha}^{p} \quad \text { sur } B \\
\int_{B} \Phi_{\alpha}^{p+1} d v(g)=1
\end{gathered}
$$

ò̀ $\Delta_{g} u=-g^{i j}\left(\partial_{i j} u-\Gamma_{i j}^{k} \partial_{k} u\right)$.

Démonstration. - L'inclusion de $H_{0,1}(B)$ dans $L^{q+1}(B)$ étant compacte pour tout $q<p$, on démontre sans difficulté l'existence de $\Phi_{q} \in$ Vol. 13, $\mathrm{n}^{\circ} 1-1996$. 
$C^{2}(\bar{B}) \cap H_{0,1}(B), \Phi_{q}>0$ sur $B$, et l'existence de $\lambda_{q}>0$ tels que

$$
\begin{gathered}
\Delta_{g} \Phi_{q}+\alpha \Phi_{q}=\lambda_{q} \Phi_{q}^{q} \quad \text { sur } B \\
\int_{B} \Phi_{q}^{q+1} d v(g)=1 \\
\lim _{q \rightarrow p} \lambda_{q}=\operatorname{Inf}_{u} I_{\alpha}(u) .
\end{gathered}
$$

Les techniques utilisées pour démontrer un tel résultat sont maintenant classiques. $\left(\Phi_{q}\right)$ étant bornée dans $H_{0,1}(B)$, on pourra en plus supposer que $\lim _{q \rightarrow p} \Phi_{q}=\Phi_{\alpha}$ faiblement dans $H_{0,1}(B)$, fortement dans $L^{2}(B)$ et presque partout. Comme $\lim _{q \rightarrow p} \lambda_{q}=\operatorname{Inf}_{u} I_{\alpha}(u)<\frac{1}{S_{N}}$, les mêmes arguments que ceux utilisés dans la démonstration du théorème 1 de [4] nous permettent alors de montrer que $\Phi_{\alpha} \not \equiv 0$.

$\Phi_{\alpha}$ devient ainsi une solution faible, positive et non identiquement nulle, de l'équation

$$
\Delta_{g} u+\alpha u=\left(\operatorname{Inf}_{u} I_{\alpha}(u)\right) u^{p} .
$$

Par théorie classique de régularité, on obtient maintenant $\Phi_{\alpha} \in C^{2}(\bar{B})$, et avec le principe du maximum, on obtient $\Phi_{\alpha}>0$ sur $B$.

Il reste donc à montrer que $\int_{B} \Phi_{\alpha}^{p+1} d v(g)=1$. L'inclusion de $H_{0,1}(B)$ dans $L^{p}(B)$ étant compacte, sans perdre en généralité, on pourra supposer que $\lim _{q \rightarrow p} \Phi_{q}=\Phi_{\alpha}$ dans $L^{p}(B)$.

On obtient alors :

$$
\begin{aligned}
\int_{B} \Phi_{\alpha}^{p+1} d v(g)= & \lim _{q \rightarrow p} \int_{B} \Phi_{q}^{p} \Phi_{\alpha} d v(g) \\
\leq & \lim _{q \rightarrow p}\left(\int_{B} \Phi_{q}^{q+1} d v(g)\right)^{p /(q+1)} \\
& \times\left(\int_{B} \Phi_{\alpha}^{(q+1) /(q+1-p)} d v(g)\right)^{(q+1-p) /(q+1)} \\
\leq & \left(\int_{B} \Phi_{\alpha}^{p+1} d v(g)\right)^{1 /(p+1)} \text { puisque } \int_{B} \Phi_{q}^{q+1} d v(g)=1 .
\end{aligned}
$$

Par suite, $\int_{B} \Phi_{\alpha}^{p+1} d v(g) \leq 1$ 
Indépendamment,

$$
\int_{B}\left|\nabla_{g} \Phi_{\alpha}\right|^{2} d v(g)+\alpha \int_{B} \Phi_{\alpha}^{2} d v(g)=\left(\operatorname{Inf}_{u} I_{\alpha}(u)\right) \int_{B} \Phi_{\alpha}^{p+1} d v(g) .
$$

On obtient ainsi :

$$
\begin{aligned}
\operatorname{Inf}_{u} I_{\alpha}(u) & \leq \frac{\int_{B}\left|\nabla_{g} \Phi_{\alpha}\right|^{2} d v(g)+\alpha \int_{B} \Phi_{\alpha}^{2} d v(g)}{\left(\int_{B} \Phi_{\alpha}^{p+1} d v(g)\right)^{2 /(p+1)}} \\
& \leq\left(\operatorname{Inf}_{u} I_{\alpha}(u)\right)\left(\int_{B} \Phi_{\alpha}^{p+1} d v(g)\right)^{2 / N} \leq \operatorname{Inf}_{u} I_{\alpha}(u) .
\end{aligned}
$$

et, comme conséquence directe de ces inégalités, $\int_{B} \Phi_{\alpha}^{p+1} d v(g)=1$. La proposition est donc bien démontrée.

Ainsi, démontrer la conjecture revient à montrer que la situation décrite par la proposition 2 ne peut pas se produire.

\section{THÉORIE CLASSIQUE DES POINTS DE CONCENTRATION}

La démonstration de la conjecture se fait par l'absurde. On suppose donc que pour tout $\alpha>0$, il existe $\Phi_{\alpha} \in C^{2}(\bar{B}) \cap H_{0,1}(B), \Phi_{\alpha}>0$ sur $B$, et il existe $\left.\lambda_{\alpha} \in\right] 0, \frac{1}{S_{N}}[$, tels que :

$$
\begin{gathered}
\Delta_{g} \Phi_{\alpha}+\alpha \Phi_{\alpha}=\lambda_{\alpha} \Phi_{\alpha}^{p} \operatorname{sur} B \\
\int_{B} \Phi_{\alpha}^{p+1} d v(g)=1 .
\end{gathered}
$$

On étudie dans ce paragraphe les premières propriétés vérifiées par les $\Phi_{\alpha}$. Pour un complément sur le type des phénomènes décrits ici on pourra voir [19], [21], [22] et [25]. Dans tout ce qui suit nous considérons des suites de réels $\alpha$ qui tendent vers $+\infty$, et nous prenons successivement des sous suites.

Lemme 3. - (1) Toute sous suite de $\left(\Phi_{\alpha}\right)$ qui converge dans un $L^{q}(B), q>1$, a pour limite 0 . En particulier, on pourra supposer que $\lim _{\alpha \rightarrow \infty} \Phi_{\alpha}=0$ p.p. et dans $L^{2}(B)$. 
(2) $\lim _{\alpha \rightarrow \infty} \lambda_{\alpha}=\frac{1}{S_{N}}$.

Démonstration. - (1) Puisque

$\alpha \int_{B} \Phi_{\alpha}^{2} d v(g) \leq \int_{B}\left|\nabla_{g} \Phi_{\alpha}\right|^{2} d v(g)+\alpha \int_{B} \Phi_{\alpha}^{2} d v(g) \leq \lambda_{\alpha} \leq \frac{1}{S_{N}}$

on obtient $\lim _{\alpha \rightarrow \infty} \int_{B} \Phi_{\alpha}^{2} d v(g)=0$. La première partie du lemme est donc bien démontrée.

(2) Supposons maintenant qu'il existe une sous suite $\left(\lambda_{\alpha}\right)$ de $\left(\lambda_{\alpha}\right)$ vérifiant $\lim _{\alpha \rightarrow \infty} \lambda_{\alpha}=\lambda<\frac{1}{S_{N}}$. Soit $\varepsilon>0$ choisi de sorte que $(1+\varepsilon) \lambda<$ $\frac{1}{S_{N}}$, et soit $C_{\varepsilon}$ une constante strictement positive pour laquelle

$$
\begin{gathered}
\forall u \in H_{0,1}(B), \\
\left(\int_{B}|u|^{p+1} d v(g)\right)^{2 /(p+1)} \\
\leq(1+\varepsilon) S_{N}\left(\int_{B}\left|\nabla_{g} u\right|^{2} d v(g)+C_{\varepsilon} \int_{B} u^{2} d v(g)\right) .
\end{gathered}
$$

On a alors $\quad \lambda_{\alpha}=\int_{B}\left|\nabla_{g} \Phi_{\alpha}\right|^{2} d v(g)+\alpha \int_{B} \Phi_{\alpha}^{2} d v(g)$

$$
\geq \frac{1}{(1+\varepsilon) S_{N}}+\left(\alpha-C_{\varepsilon}\right) \int_{B} \Phi_{\alpha}^{2} d v(g)
$$

puisque $\int_{B} \Phi_{\alpha}^{p+1} d v(g)=1$

Par suite, on obtient

$$
\left(\frac{1}{S_{N}}-(1+\varepsilon) \lambda_{\alpha}\right)+(1+\varepsilon)\left(\alpha-C_{\varepsilon}\right) \int_{B} \Phi_{\alpha}^{2} d v(g) \leq 0,
$$

ce qui est impossible pour $\alpha \gg 1$. Le lemme est donc bien démontré.

LEMME 4. - $\lim _{\alpha \rightarrow \infty} \alpha \int_{B} \Phi_{\alpha}^{2} d v(g)=0$.

Démonstration. - Soit $\varepsilon>0$ et soit $C_{\varepsilon}>0$ telle que pour tout $u \in H_{0,1}(B)$,

$$
\left(\int_{B}|u|^{p+1} d v(g)\right)^{2 /(p+1)} \leq\left(S_{N}+\varepsilon\right) \int_{B}\left|\nabla_{g} u\right|^{2} d v(g)+C_{\varepsilon} \int_{B} u^{2} d v(g) .
$$


D'après (1) et (2),

$$
\int_{B}\left|\nabla_{g} \Phi_{\alpha}\right|^{2} d v(g)+\alpha \int_{B} \Phi_{\alpha}^{2} d v(g)=\lambda_{\alpha}\left(\int_{B} \Phi_{\alpha}^{p+1} d v(g)\right)^{2 /(p+1)}
$$

Par suite,

$$
\begin{aligned}
\int_{B}\left|\nabla_{g} \Phi_{\alpha}\right|^{2} d v(g)+\alpha \int_{B} \Phi_{\alpha}^{2} d v(g) \leq & \lambda_{\alpha}\left(S_{N}+\varepsilon\right) \int_{B}\left|\nabla_{g} \Phi_{\alpha}\right|^{2} d v(g) \\
& +\lambda_{\alpha} C_{\varepsilon} \int_{B} \Phi_{\alpha}^{2} d v(g) .
\end{aligned}
$$

Comme par ailleurs, $\lambda_{\alpha}<\frac{1}{S_{N}}$ et $\int_{B}\left|\nabla_{g} \Phi_{\alpha}\right|^{2} d v(g) \leq \lambda_{\alpha}$, on obtient l'existence d'une constante $C>0$, indépendante de $\alpha$ et $\varepsilon$, pour laquelle

$$
\alpha \int_{B} \Phi_{\alpha}^{2} d v(g) \leq C \varepsilon+\tilde{C}_{\varepsilon} \int_{B} \Phi_{\alpha}^{2} d v(g) .
$$

Du lemme 3 on déduit alors $\varlimsup_{\alpha \rightarrow \infty} \alpha \int_{B} \Phi_{\alpha}^{2} d v(g) \leq C \varepsilon . \varepsilon$ étant arbitraire, on récupère $\lim _{\alpha \rightarrow \infty} \alpha \int_{B} \Phi_{\alpha}^{2} d v(g)=0$, et le lemme est démontré.

Comme dans [13], on dira maintenant que $x_{0} \in \bar{B}$ est un point de concentration de $\left(\Phi_{\alpha}\right)$ si pour tout $\delta>0, \varlimsup_{\alpha \rightarrow \infty} \int_{B\left(x_{0}, \delta\right)} \Phi_{\alpha}^{p+1} d v(g)>0$. (Une fois pour toutes, par l'intégrale sur $B\left(x_{0}, \delta\right)$ on entend l'intégrale sur $B\left(x_{0}, \delta\right) \cap \bar{B}$.) On démontre alors le résultat suivant.

LEMME 5. - (1) Si $x_{0}$ est un point de concentration de $\left(\Phi_{\alpha}\right)$, alors pour tout $\delta>0, \varlimsup_{\alpha \rightarrow \infty} \int_{B\left(x_{0}, \delta\right)} \Phi_{\alpha}^{p+1} d v(g)=1$

(2) $\left(\Phi_{\alpha}\right)$ possède un et un seul point de concentration (toujours quitte à extraire une sous suite)

(3) $\lim _{\alpha \rightarrow \infty}\left\|\Phi_{\alpha}\right\|_{L^{\infty}(B)}=\infty$.

Démonstration. - (1) Soit $x_{0} \in \bar{B}$ et soit $\eta \in C^{\infty}\left(\mathbb{R}^{N}\right)$ vérifiant $0 \leq \eta \leq 1, \eta=1$ sur $B\left(x_{0}, \delta / 2\right), \eta=0$ sur $\mathbb{R}^{N} \backslash B\left(x_{0}, \delta\right), \delta>0$. 
En multipliant (1) par $\eta^{2} \Phi_{\alpha}^{k}, k \geq 1$, on obtient (voir [14]) :

$$
\begin{aligned}
& \frac{4 k}{(k+1)^{2}} \int_{B}\left|\nabla_{g}\left(\eta \Phi_{\alpha}^{(k+1) / 2}\right)\right|^{2} d v(g) \\
& \quad-\frac{2(k-1)}{(k+1)^{2}} \int_{B} \eta\left(\Delta_{g} \eta\right) \Phi_{\alpha}^{k+1} d v(g) \\
& \quad-\frac{2}{k+1} \int_{B}\left|\nabla_{g} \eta\right|^{2} \Phi_{\alpha}^{k+1} d v(g)+\alpha \int_{B} \eta^{2} \Phi_{\alpha}^{k+1} d v(g) \\
& =\lambda_{\alpha} \int_{B} \eta^{2} \Phi_{\alpha}^{k+p} d v(g) .
\end{aligned}
$$

Les inégalités de Hölder pour le membre de droite de (3), l'inégalité de Sobolev classique rappelée dans l'introduction et le fait que $\left(\Phi_{\alpha}\right)$ soit bornée dans $H_{1}$, permettent alors de montrer le résultat suivant :

$$
\begin{gathered}
\forall \varepsilon>0, \exists C_{1}>0 / \forall \delta \ll 1 \text { et } \forall k \in[1, p], \\
\int_{B}\left|\nabla_{g}\left(\eta \Phi_{\alpha}^{(k+1) / 2}\right)\right|^{2} d v(g) \\
\leq \frac{(k+1)^{2}}{4 k} \lambda_{\alpha}\left(S_{N}+\varepsilon\right)\left(\int_{B\left(x_{0}, \delta\right)} \Phi_{\alpha}^{p+1} d v(g)\right)^{(p-1)(p+1)} \\
\quad \times \int_{B}\left|\nabla_{g}\left(\eta \Phi_{\alpha}^{(k+1) / 2}\right)\right|^{2} d v(g)+C_{1} .
\end{gathered}
$$

Par suite, en tenant compte du fait que $\lim _{\alpha \rightarrow \infty} \lambda_{\alpha}=\frac{1}{S_{N}}$, on obtient :

(4)

$$
\begin{aligned}
\forall \varepsilon>0, \exists C_{1}>0 / \forall \delta \ll 1, \forall \alpha \gg 1 \text { et } \forall k \in[1, p], \\
\int_{B}\left|\nabla_{g}\left(\eta \Phi_{\alpha}^{(k+1) / 2}\right)\right|^{2} d v(g) \\
\leq \frac{(k+1)^{2}}{4 k}(1+\varepsilon)\left(\int_{B\left(x_{0}, \delta\right)} \Phi_{\alpha}^{p+1} d v(g)\right)^{(p-1)(p+1)} \\
\quad \times \int_{B}\left|\nabla_{g}\left(\eta \Phi_{\alpha}^{(k+1) / 2}\right)\right|^{2} d v(g)+C_{1} .
\end{aligned}
$$

Supposons maintenant qu'il existe $\delta_{0}>0$ tel que

$$
\varlimsup_{\alpha \rightarrow \infty} \int_{B\left(x_{0}, \delta_{0}\right)} \Phi_{\alpha}^{p+1} d v(g)<1 .
$$


En utilisant (4), on obtient pour $k>1$ suffisamment proche de 1 et pour $\delta \ll 1$,

$$
\int_{B}\left|\nabla_{g}\left(\eta \Phi_{\alpha}^{(k+1) / 2}\right)\right|^{2} d v(g) \leq C_{2} \int_{B}\left|\nabla_{g}\left(\eta \Phi_{\alpha}^{(k+1) / 2}\right)\right|^{2} d v(g)+C_{3},
$$

où $C_{2}$ et $C_{3}$ sont deux constantes indépendantes de $\alpha$ avec $C_{2}<1$.

Par suite, $\int_{B}\left|\nabla_{g}\left(\eta \Phi_{\alpha}^{(k+1) / 2}\right)\right|^{2} d v(g) \leq$ Cte.

Posons alors $a=\varlimsup_{\alpha \rightarrow \infty} \int_{B\left(x_{0}, \delta_{0} / 2\right)} \Phi_{\alpha}^{p+1} d v(g)$. Par définition, si $x_{0}$ est un point de concentration de $\left(\Phi_{\alpha}\right), a>0$.

Indépendamment, avec Hölder,

$$
\begin{aligned}
& \int_{B\left(x_{0}, \delta_{0} / 2\right)} \Phi_{\alpha}^{p+1} d v(g) \\
& \leq\left(\int_{B\left(x_{0}, \delta_{0} / 2\right)} \Phi_{\alpha}^{N(k+1) /(N-2)} d v(g)\right)^{(N+2) / N(k+1)} \\
& \quad\left(\int_{B\left(x_{0}, \delta_{0} / 2\right)} \Phi_{\alpha}^{N(k+1) /(N k-2)} d v(g)\right)^{(N k-2) / N(k+1)} \\
& \leq C_{4}\left(\int_{B\left(x_{0}, \delta_{0} / 2\right)} \Phi_{\alpha}^{N(k+1) /(N k-2)} d v(g)\right)^{(N k-2) / N(k+1)}
\end{aligned}
$$

puisque

$$
\int_{B}\left|\nabla_{g}\left(\eta \Phi_{\alpha}^{(k+1) / 2}\right)\right|^{2} d v(g) \leq \text { Cte. }
$$

Par conséquent, si $k_{1}=\frac{N(k+1)}{N k-2}$, on obtient

$$
1<k_{1}<p+1 \quad \text { et } \quad \int_{B} \Phi_{\alpha}^{k_{1}} d v(g) \geq C_{5}>0 .
$$

Cette dernière inégalité contredisant le premier point du lemme 3 , la première partie du lemme 5 est démontrée.

(2) En vertu de ce qui vient d'être dit, et puisque $\int_{B} \Phi_{\alpha}^{p+1} d v(g)=1$, quitte à extraire une sous suite $\left(\Phi_{\alpha}\right)$ possède au plus un point de concentration. À l'inverse, par définition même des points de concentration, on montre facilement que $\left(\Phi_{\alpha}\right)$ possède au moins un point de concentration. La seconde partie du lemme 5 est donc démontrée. 
(3) Pour finir, $\lim _{\alpha \rightarrow \infty}\left\|\Phi_{\alpha}\right\|_{L^{\infty}(B)}=\infty$ se démontre avec la première partie du lemme 5. Il suffit de remarquer que pour tout $\delta>0$, $\int_{B\left(x_{0}, \delta\right)} \Phi_{\alpha}^{p+1} d v(g) \leq \operatorname{Cte} \delta^{N}\left\|\Phi_{\alpha}\right\|_{L^{\infty}(B)}^{p+1}$.

LEMME 6. - Soit $x_{0}$ le point de concentration de $\left(\Phi_{\alpha}\right)$. Alors, $\lim _{\alpha \rightarrow \infty} \Phi_{\alpha}=0$ dans $C_{\text {loc }}^{1}\left(\bar{B} \backslash\left\{x_{0}\right\}\right)$.

Démonstration. - Soit $x \neq x_{0}, x \in \bar{B}$. Pour $\delta \ll 1$ on a alors $\varlimsup_{\alpha \rightarrow \infty} \int_{B(x, \delta)} \Phi_{\alpha}^{p+1} d v(g)=0$. Par ailleurs, voir (3) et (4),

(5) $\int_{B}\left|\nabla_{g}\left(\eta \Phi_{\alpha}^{(k+1) / 2}\right)\right|^{2} d v(g) \leq C_{1} \int_{B}\left|\nabla_{g}\left(\eta \Phi_{\alpha}^{(k+1) / 2}\right)\right|^{2} d v(g)+C_{2}$

où

$$
\begin{aligned}
C_{1} & =\frac{(k+1)^{2}}{4 k}(1+\varepsilon)\left(\int_{B(x, \delta)} \Phi_{\alpha}^{p+1} d v(g)\right)^{(p-1) /(p+1)} \\
C_{2} & \leq \frac{(k-1)}{2 k} \int_{B} \eta\left|\Delta_{g} \eta\right| \Phi_{\alpha}^{k+1} d v(g)+\frac{(k+1)}{2 k} \int_{B}\left|\nabla_{g} \eta\right|^{2} \Phi_{\alpha}^{k+1} d v(g) .
\end{aligned}
$$

Par suite :

(i) $\lim _{\alpha \rightarrow \infty} \int_{B}\left|\nabla_{g}\left(\eta \Phi_{\alpha}^{(k+1) / 2}\right)\right|^{2} d v(g)=0$ pour tout $1 \leq k \leq p$

(ii) $\lim _{\alpha \rightarrow \infty} \int_{B(x, \delta / 2)} \Phi_{\alpha}^{(k+1)(p+1) / 2} d v(g)=0$ pour tout $1 \leq k \leq p$ (avec Sobolev).

(ii) nous permet maintenant d'utiliser (5) avec $k=\frac{(p+1)^{2}}{2}-1$, et on obtient ainsi $\lim _{\alpha \rightarrow \infty} \int_{B\left(x, \delta^{\prime}\right)} \Phi_{\alpha}^{(p+1)^{3} / 4} d v(g)=0$ pour un certain $\delta^{\prime}>0$, $\delta^{\prime} \ll 1$.

Finalement, par récurrence, on voit que pour tout $x \neq x_{0}$, et tout $q$, il existe $\delta \ll 1$ tel que $\lim _{\alpha \rightarrow \infty} \int_{B(x, \delta)} \Phi_{\alpha}^{q} d v(g)=0$. Par suite, puisque $\Delta_{g} \Phi_{\alpha} \leq \lambda_{\alpha} \Phi_{\alpha}^{p}$, on obtient que pour tout $\omega \Subset \bar{B} \backslash\left\{x_{0}\right\}, \lim _{\alpha \rightarrow \infty} \Phi_{\alpha}=0$ dans $L^{\infty}(\omega)$. (Voir [11], théorème 8.25.)

Pour finir, le lemme 7 ci-dessous, le théorème 8.32 et le corollaire 8.36 de [11], montrent que $\lim _{\alpha \rightarrow \infty} \Phi_{\alpha}=0$ dans $C_{\text {loc }}^{1}\left(\bar{B} \backslash\left\{x_{0}\right\}\right)$.

Lemme 7. - Soit $x_{0}$ le point de concentration de $\left(\Phi_{\alpha}\right)$. Alors, pour tout $q$ et tout $\omega \Subset \bar{B} \backslash\left\{x_{0}\right\}, \lim _{\alpha \rightarrow \infty} \alpha^{q}\left\|\Phi_{\alpha}\right\|_{L^{\infty}(\omega)}=0$. En particulier, pour tout $\omega \Subset \bar{B} \backslash\left\{x_{0}\right\}, \lim _{\alpha \rightarrow \infty} \alpha\left\|\Phi_{\alpha}\right\|_{L^{\infty}(\omega)}=0$. 
Démonstration. - Soit $x \neq x_{0}, x \in \bar{B}$, et soit $\delta>0$ tel que $x_{0} \notin \bar{B}(x, \delta)$. On a alors, voir (3),

$$
\begin{aligned}
\alpha^{q+1} \int_{B} \eta^{2} \Phi_{\alpha}^{k+1} d v(g) \leq & C_{1} \alpha^{q} \int_{B(x, \delta)} \Phi_{\alpha}^{k+1} d v(g) \\
& +C_{2} \alpha^{q} \int_{B(x, \delta)} \Phi_{\alpha}^{k+p} d v(g),
\end{aligned}
$$

où $C_{1}$ et $C_{2}$ ne dépendent que de $\eta$ et $k$.

Par ailleurs, en raison de ce qui vient d'être démontré, pour tout $\omega \Subset \bar{B} \backslash\left\{x_{0}\right\}$ et tout $m, \lim _{\alpha \rightarrow \infty} \int_{\omega} \Phi_{\alpha}^{m} d v(g)=0$. Par suite, en raisonnant par récurrence sur $q$, on obtient que pour tout $x \neq x_{0}$, tout $m$ et tout $q$, il existe $\delta>0, \delta \ll 1$, tel que

$$
\lim _{\alpha \rightarrow \infty} \alpha^{q} \int_{B(x, \delta)} \Phi_{\alpha}^{m} d v(g)=0 .
$$

Pour finir, comme $\Delta_{g} \Phi_{\alpha} \leq \lambda_{\alpha} \Phi_{\alpha}^{p}$, on obtient que pour tout $q$ et tout $\omega \Subset \bar{B} \backslash\left\{x_{0}\right\}, \lim _{\alpha \rightarrow \infty} \alpha^{q}\left\|\Phi_{\alpha}\right\|_{L^{\infty}(\omega)}=0$. (Voir [11], théorème 8.25).

Le lemme est donc bien démontré.

On note alors

$$
u_{\alpha}=\left(\frac{\lambda_{\alpha}}{N(N-2)}\right)^{(N-2) / 4} \Phi_{\alpha} .
$$

Tout comme les $\Phi_{\alpha}$, les $u_{\alpha}$ se concentrent au point $x_{0}$, et :

$$
\begin{gathered}
\Delta_{g} u_{\alpha}+\alpha u_{\alpha}=N(N-2) u_{\alpha}^{p} \operatorname{sur} B \\
\lim _{\alpha \rightarrow \infty} \int_{B} u_{\alpha}^{p+1} d v(g)=\frac{\omega_{N}}{2^{N}} \\
\lim _{\alpha \rightarrow \infty} \int_{B}\left|\nabla_{g} u_{\alpha}\right|^{2} d v(g)=\frac{N(N-2) \omega_{N}}{2^{N}} .
\end{gathered}
$$

Soit maintenant $x_{\alpha}$ un point de $B$ tel que

$$
u_{\alpha}\left(x_{\alpha}\right)=\left\|u_{\alpha}\right\|_{L^{\infty}(B)}
$$

et soit $\mu_{\alpha}$ tel que

$$
\left\|u_{\alpha}\right\|_{L^{\infty}(B)}=\mu_{\alpha}^{-(N-2) / 2} .
$$

D'après les lemmes 5 et $6, \lim _{\alpha \rightarrow \infty} x_{\alpha}=x_{0}$ et $\lim _{\alpha \rightarrow \infty} \mu_{\alpha}=0$. De plus :

LEMME 8. - $\left(\alpha \mu_{\alpha}^{2}\right)$ est bornée.

Démonstration. - Au point $x_{\alpha}, \Delta_{g} u_{\alpha} \geq 0$. Par suite, $\alpha \mu_{\alpha}^{-(N-2) / 2} \leq$ $N(N-2) \mu_{\alpha}^{-(N+2) / 2}$, et $\alpha \mu_{\alpha}^{2} \leq N(N-2)$. 


\section{ESTIMATION DE LA VITESSE DE CONVERGENCE DES $x_{\alpha}$ AU BORD DE $B$}

On note $d$ la distance euclidienne de $\mathbb{R}^{N}$. Les $x_{\alpha}$ ayant été introduits à la fin du paragraphe 4 , l'objet de cette partie est de démontrer la proposition suivante.

Proposition 9. $-\lim _{\alpha \rightarrow \infty} \frac{d\left(x_{\alpha}, \partial B\right)}{\mu_{\alpha}}=+\infty$.

Démonstration. - Puisque $\lim _{\alpha \rightarrow \infty} x_{\alpha}=x_{0}$, on remarque déjà que le résultat est immédiat si $x_{0} \in B$. Sans perdre en généralité, on pourra donc supposer que $x_{0}=(0, \ldots, 0,1) \in \partial B$. On introduit maintenant les isométries vectorielles de $\mathbb{R}^{N}$ qui ramènent le point $x_{\alpha}$ sur un point $x_{\alpha}^{R} \in\left[0, x_{0}\right]$ (où $\left.\left[0, x_{0}\right]=\left\{\theta x_{0}, 0 \leq \theta \leq 1\right\}\right)$. En composant $g$ et $u_{\alpha}$ par ces isométries, on obtient une famille $g_{\alpha}$ de métriques sur $\bar{B}$, et des fonctions encore notées $u_{\alpha}$, qui vérifient :

$$
\begin{gathered}
\lim _{\alpha \rightarrow \infty} g_{\alpha}=g \operatorname{dans} C^{2}(\bar{B}) \\
u_{\alpha} \in C^{2}(\bar{B}) \cap H_{0,1}(B), u_{\alpha}>0 \operatorname{sur} B \\
\Delta_{g_{\alpha}} u_{\alpha}+\alpha u_{\alpha}=N(N-2) u_{\alpha}^{p} \operatorname{sur} B \\
\lim _{\alpha \rightarrow \infty} \int_{B} u_{\alpha}^{p+1} d v\left(g_{\alpha}\right)=\frac{\omega_{N}}{2^{N}} \\
\lim _{\alpha \rightarrow \infty}\left|\nabla_{g_{\alpha}} u_{\alpha}\right|^{2} d v\left(g_{\alpha}\right)=\frac{N(N-2) \omega_{N}}{2^{N}} \\
u_{\alpha}\left(x_{\alpha}^{R}\right)=\mu_{\alpha}^{-(N-2) / 2}=\left\|u_{\alpha}\right\|_{L^{\infty}(B)} .
\end{gathered}
$$

Comme $d\left(x_{\alpha}, \partial B\right)=d\left(x_{\alpha}^{R}, x_{0}\right)$, il nous faut montrer que $\lim _{\alpha \rightarrow \infty} \frac{d\left(x_{\alpha}^{R}, x_{0}\right)}{\mu_{\alpha}}=\infty$. Pour se faire, on introduit les fonctions

$$
v_{\alpha}(x)=\mu_{\alpha}^{(N-2) / 2} u_{\alpha}\left(\mu_{\alpha} x+x_{0}\right) .
$$

$v_{\alpha}$ est définie pour $x \in B_{\alpha}=B\left(\frac{-x_{0}}{\mu_{\alpha}}, \frac{1}{\mu_{\alpha}}\right)$. On a alors $0 \leq v_{\alpha} \leq 1$ et $\cup B_{\alpha}=E$ où $E=\left\{\left(x_{1}, \ldots, x_{N}\right) \in \mathbb{R}^{N} / x_{N}<0\right\}$. De plus, $0 \in \bar{B}_{\alpha}$ pour tout $\alpha$, et si on suppose que $\left(\mu_{\alpha}\right)$ est décroissante (ce qui ne nuit pas à la généralité), on obtient $B_{\alpha} \subset B_{\alpha^{\prime}}$ dès que $\alpha<\alpha^{\prime}$. 
On définit maintenant $h_{\alpha}(x)=g_{\alpha}\left(\mu_{\alpha} x+x_{0}\right), x \in B_{\alpha}$. D'après (9), pour tout $K \Subset E, \lim _{\alpha \rightarrow \infty} h_{\alpha}=g\left(x_{0}\right)$ dans $C^{2}(K)$. De plus, si $\xi$ désigne la métrique euclidienne de $\mathbb{R}^{N}$ :

$$
\exists \lambda>0 / \forall \alpha, \quad \frac{1}{\lambda} \xi \leq h_{\alpha} \leq \lambda \xi
$$

les $h_{\alpha}$ sont uniformément bornées $C^{1} \operatorname{sur} B_{\alpha}$.

Par ailleurs, avec (11), on voit facilement que

$$
\Delta_{h_{\alpha}} v_{\alpha}+\left(\alpha \mu_{\alpha}^{2}\right) v_{\alpha}=N(N-2) v_{\alpha}^{p} \operatorname{sur} B_{\alpha} .
$$

On définit alors $y_{\alpha}=\frac{\left(x_{\alpha}^{R}-x_{0}\right)}{\mu_{\alpha}} \cdot y_{\alpha} \in B_{\alpha}$ et $v_{\alpha}\left(y_{\alpha}\right)=1$.

On distingue maintenant deux étapes dans la démonstration.

Étape 1. - On montre que $\varliminf_{\alpha \rightarrow \infty} \frac{d\left(x_{\alpha}^{R}, x_{0}\right)}{\mu_{\alpha}}=0$ est impossible. Si ce n'était pas le cas, quitte à extraire une sous suite, on pourrait supposer que $\lim _{\alpha \rightarrow \infty} \frac{d\left(x_{\alpha}^{R}, x_{0}\right)}{\mu_{\alpha}}=0$. Par suite, on aurait $\lim _{\alpha \rightarrow \infty} y_{\alpha}=0$. Or, voir l'appendice 2, les relations (15), (16), (17), le lemme 8 et le fait que $0 \leq v_{\alpha} \leq 1$, entraînent que les $v_{\alpha}$ sont uniformément bornées $C^{1}$ au voisinage de 0 . Avec le théorème des accroissement finis, on devrait donc avoir

$$
1=\left|v_{\alpha}\left(y_{\alpha}\right)-v_{\alpha}(0)\right| \leq \operatorname{Cte} d\left(0, y_{\alpha}\right)
$$

ce qui est impossible. En conclusion, $\varliminf_{\alpha \rightarrow \infty} \frac{d\left(x_{\alpha}^{R}, x_{0}\right)}{\mu_{\alpha}}>0$.

Étape 2. - On montre que $\varliminf_{\alpha \rightarrow \infty} \frac{d\left(x_{\alpha}^{R}, x_{0}\right)}{\mu_{\alpha}}=\ell, \ell \in \mathbb{R}, \ell>0$, est impossible. Là encore, si ce n'êtait pas le cas, quitte à extraire une sous suite, on pourrait supposer que $\lim _{\alpha \rightarrow \infty} y_{\alpha}=y_{0} \in E$. On remarque alors que $\left(v_{\alpha}\right)$ est équicontinue sur tout compact de $E$. Pour le voir, on utilise (15), (16), (17), le fait que $0 \leq v_{\alpha} \leq 1$ et le théorème 8.32 de [11]. Par suite, il existe $v \in C^{0}(E)$ telle que pour tout $K \Subset E$, une sous suite de $\left(v_{\alpha}\right)$ converge vers $v$ dans $L^{\infty}(K)$. La fonction $v$ vérifie ainsi $0 \leq v \leq 1$ et $v\left(y_{0}\right)=1$. En particulier, $v \not \equiv 0$. 
On remarque maintenant qu'à extraction près d'une sous suite, $\lim _{\alpha \rightarrow \infty} \alpha \mu_{\alpha}^{2}=0$. En effet,

$$
\alpha \int_{B} u_{\alpha}^{2} d v\left(g_{\alpha}\right)=\alpha \mu_{\alpha}^{2} \int_{B_{\alpha}} v_{\alpha}^{2} d v\left(h_{\alpha}\right) \geq \alpha \mu_{\alpha}^{2} \int_{K} v_{\alpha}^{2} d v\left(h_{\alpha}\right)
$$

Pour tout $K \Subset E$. Or,

$$
\int_{B} u_{\alpha}^{2} d v\left(g_{\alpha}\right)=\left(\frac{\lambda_{\alpha}}{N(N-2)}\right)^{(N-2) / 2} \int_{B} \Phi_{\alpha}^{2} d v\left(g_{\alpha}\right),
$$

et puisque $v \not \equiv 0$, on déduit du lemme 4 que, quitte à extraire une sous suite, $\lim _{\alpha \rightarrow \infty} \alpha \mu_{\alpha}^{2}=0$.

Par conséquent, avec (17), $v \in C^{\infty}(E)$ et

$$
\Delta_{g\left(x_{0}\right)} v=N(N-2) v^{p} \operatorname{sur} E .
$$

(On rappelle que $\lim _{\alpha \rightarrow \infty} h_{\alpha}=g\left(x_{0}\right)$ dans $C^{2}(K)$ pour tout $K \Subset E$.)

On remarque maintenant qu'en transportant la métrique $g\left(x_{0}\right)$ par un isomorphisme vectoriel de $\mathbb{R}^{N}$ convenablement choisi, et en composant $v$ par ce même isomorphisme, on obtient une solution de $\Delta_{\xi} v=N(N-2) v^{p}$ sur un demi-espace de $\mathbb{R}^{N}$. (Quitte à composer de nouveau par une isométrie vectorielle, on pourra même supposer que ce demi-espace est encore le demi-espace $E$.) Pour ne pas trop alourdir les notations, on continue de noter $v$ cette solution et $E$ le demi-espace sur lequel elle est définie.

Soit maintenant

$$
w(x)=\left(\frac{1}{1+|x|^{2}}\right)^{(N-2) / 2},|x|=d(0, x) .
$$

On pose $h=w^{4 /(N-2)} \xi$. À un facteur multiplicatif 4 près, $h$ représente la métrique standard de la sphère après projection stéréographique. On note alors $\tilde{v}=\frac{v}{w}$ et $\tilde{v}_{\alpha}=\frac{v_{\alpha}}{w}$. La courbure scalaire de la métrique $h$ valant $4 N(N-1)$, on obtient

$$
\Delta_{h} \tilde{v}+N(N-2) \tilde{v}=N(N-2) \tilde{v}^{p} \quad \text { sur } E .
$$


De plus,

$$
\begin{array}{ll}
\int_{B_{\alpha}}\left|\nabla_{h} \tilde{v}_{\alpha}\right|^{2} d v(h)+N(N-2) \int_{B_{\alpha}} \tilde{v}_{\alpha}^{2} d v(h) \\
=\int_{B_{\alpha}}\left|\nabla_{\xi} v_{\alpha}\right|^{2} d v(\xi) & \\
\leq C_{1} \int_{B_{\alpha}}\left|\nabla_{h_{\alpha}} v_{\alpha}\right|^{2} d v\left(h_{\alpha}\right) & \text { (d'après }(15)) \\
\leq C_{2} \int_{B_{\alpha}} v_{\alpha}^{p+1} d v\left(h_{\alpha}\right) & \text { (d'après }(17)) \\
=C_{2} \int_{B} u_{\alpha}^{p+1} d v\left(g_{\alpha}\right) & \\
\leq \text { Cte } & \text { (d'après }(12)) .
\end{array}
$$

Les $\left(\tilde{v}_{\alpha}\right)$ sont donc uniformément bornées dans $H_{0,1}(E, h)$. Par suite, puisque $\lim _{\alpha \rightarrow \infty} \tilde{v}_{\alpha}=\tilde{v}$ uniformément sur tout compact de $E$, on obtient $\tilde{v} \in H_{0,1}(E, h)$. (Toute suite bornée dans un Hilbert possède une sous suite faiblement convergente.)

Notons maintenant $S^{N}$ la sphère unité de $\mathbb{R}^{N+1}$ et $g_{0}$ sa métrique standard. Par projection stéréographique, $E$ devient une demi-sphère $S^{+}$de $S^{N}$. Avec (19) on obtient ainsi une solution positive $v_{1} \in H_{0,1}\left(S^{+}, g_{0}\right)$ de

$$
\Delta_{g_{0}} v_{1}+\frac{N(N-2)}{4} v_{1}=\frac{N(N-2)}{4} v_{1}^{p} \operatorname{sur} S^{+} \text {. }
$$

En effectuant une nouvelle projection stéréographique, mais de pôle opposé au sommet de $S^{+}$, on obtient alors une solution positive $v_{2} \in$ $H_{0,1}(B)$ de

$$
\Delta_{\xi} v_{2}=\frac{N(N-2)}{4} v_{2}^{p} \operatorname{sur} B
$$

Or ceci est impossible d'après Pohozaev. On obtient donc la contradiction recherchée, et, par suite, $\lim _{\alpha \rightarrow \infty} \frac{d\left(x_{\alpha}^{R}, x_{0}\right)}{\mu_{\alpha}}=\infty$. En conclusion, la proposition 9 est démontrée.

(On rappelle que $d\left(x_{\alpha}^{R}, x_{0}\right)=d\left(x_{\alpha}, \partial B\right)$.)

Remarque. - Avec les résultats de [10], théorème 2, $x_{0}$ se trouve forcément dans $B$ si la métrique $g$ est euclidienne au voisinage de $\partial B$. Dans ce cas particulier, la proposition 9 est immédiate. 


\section{TRANSFORMATION DU PROBLÈME ET BLOW-UP}

La métrique $g$ vérifiant la propriété $(*)$ sur $\bar{B}$, il existe une boule ouverte $B^{\prime}$, centrée en 0 et contenant $\bar{B}$, telle que $g$ soit géodésiquement convexe sur $B$ et sur $B^{\prime}$. L'application exponentielle est alors une fonction $C^{\infty}$ de deux variables, à valeurs dans $B^{\prime}$, et définie sur un voisinage ouvert de $B^{\prime} \times\{0\}$ dans $B^{\prime} \times \mathbb{R}^{N}$. (Pour plus de détails, voir [17], tome 1.) Par ailleurs, puisque la métrique $g$ est géodésiquement convexe sur $B^{\prime}$, pour tout $x$ donné de $B^{\prime}, \exp _{x}$ réalise un $C^{\infty}$ difféomorphisme d'un voisinage ouvert de 0 dans $\mathbb{R}^{N}$ sur $B^{\prime}$. Avec le théorème des fonctions implicites, $\left(\exp _{x}\right)^{-1}$, regardée comme une fonction définie sur $B^{\prime} \times B^{\prime}$, est alors $C^{\infty}$ des deux variables.

On note maintenant $\Psi_{\alpha}=\exp _{x_{\alpha}}$. $\Psi_{\alpha}$ est donc un $C^{\infty}$ difféomorphisme d'un voisinage ouvert de 0 dans $\mathbb{R}^{N}$ sur $B^{\prime}$. On note alors

$$
\begin{aligned}
\omega_{\alpha} & =\left(\Psi_{\alpha}\right)^{-1}(B) \\
g_{\alpha} & =\left(\Psi_{\alpha}\right)^{*} g \\
\tilde{u}_{\alpha} & =u_{\alpha} \circ \Psi_{\alpha} .
\end{aligned}
$$

( $u_{\alpha}$ et $x_{\alpha}$ ont été introduits à la fin du paragraphe 4).

$\omega_{\alpha}$ est un domaine relativement compact étoilé en 0 de $\mathbb{R}^{N}$ (au sens où pour tout $x$ de $\omega_{\alpha}$ et tout $0 \leq \theta \leq 1, \theta x \in \omega_{\alpha}$ ). De plus, puisque l'exponentielle et son inverse sont $C^{\infty}$ des deux variables, on obtient facilement :

$$
\exists \lambda>0 / \forall \alpha, \frac{1}{\lambda} \xi \leq g_{\alpha} \leq \lambda \xi \quad \text { ( } \xi \text { la métrique euclidienne) }
$$

$$
\lim _{\alpha \rightarrow \infty} g_{\alpha}=\left(\exp _{x_{0}}\right)^{*} g \text { dans } C^{1}(K) \text { pour tout } K \Subset\left(\exp _{x_{0}}\right)^{-1}\left(B^{\prime}\right) .
$$

Par ailleurs, toujours d'après les propriétés élémentaires de l'exponentielle, on obtient :

(23) les $g_{\alpha}$ sont géodésiquement normales en 0 . En particulier, pour tout $\alpha$ et tous $i, j, k=1, \ldots, N, g_{\alpha}(0)_{i j}=\delta_{i j}$

$$
\text { et }\left(\partial_{k} g_{\alpha}\right)(0)_{i j}=0 \text {. }
$$


Enfin :

$$
\begin{aligned}
& \tilde{u}_{\alpha} \in C^{2}\left(\bar{\omega}_{\alpha}\right) \cap H_{0,1}\left(\omega_{\alpha}\right), \tilde{u}_{\alpha}>0 \operatorname{sur} \omega_{\alpha}, \\
& \text { et } \tilde{u}_{\alpha} \text { atteint son maximum } \mu_{\alpha}^{-(N-2) / 2} \text { en } 0
\end{aligned}
$$

$$
\begin{gathered}
\Delta_{g_{\alpha}} \tilde{u}_{\alpha}+\alpha \tilde{u}_{\alpha}=N(N-2) \tilde{u}_{\alpha}^{p} \operatorname{sur} \omega_{\alpha} \\
\lim _{\alpha \rightarrow \infty} \int_{\omega_{\alpha}} \tilde{u}_{\alpha}^{p+1} d v\left(g_{\alpha}\right)=\frac{\omega_{N}}{2^{N}} \\
\lim _{\alpha \rightarrow \infty} \int_{\omega_{\alpha}}\left|\nabla_{g_{\alpha}} \tilde{u}_{\alpha}\right|^{2} d v\left(g_{\alpha}\right)=\frac{N(N-2) \omega_{N}}{2^{N}} .
\end{gathered}
$$

On définit maintenant :

$$
\begin{aligned}
& \tilde{v}_{\alpha}(x)=\mu_{\alpha}^{(N-2) / 2} \tilde{u}_{\alpha}\left(\mu_{\alpha} x\right) \\
& h_{\alpha}(x)=g_{\alpha}\left(\mu_{\alpha} x\right), x \in \Omega_{\alpha}=\frac{\omega_{\alpha}}{\mu_{\alpha}} .
\end{aligned}
$$

$\tilde{v}_{\alpha}$ vérifie alors :

$$
0 \leq \tilde{v}_{\alpha} \leq 1 \text { et } \tilde{v}_{\alpha}(0)=1
$$

$$
\Delta_{h_{\alpha}} \tilde{v}_{\alpha}+\left(\alpha \mu_{\alpha}^{2}\right) \tilde{v}_{\alpha}=N(N-2) \tilde{v}_{\alpha}^{p} \quad \text { sur } \Omega_{\alpha}
$$

Par ailleurs, comme $\lim _{\alpha \rightarrow \infty} \frac{d\left(x_{\alpha}, \partial B\right)}{\mu_{\alpha}}=\infty$ (voir proposition 9), et puisque les métriques $g$ et $g_{\alpha}$ sont équivalentes à la métrique euclidienne, on obtient $\lim _{\alpha \rightarrow \infty} d\left(0, \partial \Omega_{\alpha}\right)=\infty$. Par suite, $\cup \Omega_{\alpha}=\mathbb{R}^{N}$ au sens où pour tout $K \Subset \mathbb{R}^{N}$ et tout $\alpha \gg 1, K \subset \Omega_{\alpha}$.

Enfin, utilisant (22), on obtient

$$
\text { pour tout } K \Subset \mathbb{R}^{N}, \lim _{\alpha \rightarrow \infty} h_{\alpha}=\xi \operatorname{dans} C^{1}(K) \text {. }
$$

On étudie dans ce paragraphe les premières propriétés vérifiées par les $\tilde{v}_{\alpha}$.

Pour commencer, on remarque que (20), (28), (29), (30) et [11], théorème 8.32 , entraînent que les $\tilde{v}_{\alpha}$ sont équicontinues sur tout compact de $\mathbb{R}^{N}$. Par suite, il existe $\tilde{v} \in C^{0}\left(\mathbb{R}^{N}\right)$ telle que pour tout $K \Subset \mathbb{R}^{N}$, une sous suite de $\left(\tilde{v}_{\alpha}\right)$ converge vers $\tilde{v}$ dans $L^{\infty}(K)$. On a alors $0 \leq \tilde{v}_{\alpha} \leq 1$ et $\tilde{v}(0)=1$. En particulier, $\tilde{v} \not \equiv 0$. On démontre maintenant le résultat suivant. 
LEMME 10. - À extraction près d'une sous suite, $\lim _{\alpha \rightarrow \infty} \alpha \mu_{\alpha}^{2}=0$.

Démonstration. - On remarque que pour tout $K \Subset \mathbb{R}^{N}$,

$$
\begin{aligned}
\alpha \int_{B} u_{\alpha}^{2} d v(g)=\alpha \int_{\omega_{\alpha}} \tilde{u}_{\alpha}^{2} d v\left(g_{\alpha}\right) & =\alpha \mu_{\alpha}^{2} \int_{\Omega_{\alpha}} \tilde{v}_{\alpha}^{2} d v\left(h_{\alpha}\right) \\
& \geq \alpha \mu_{\alpha}^{2} \int_{K} \tilde{v}_{\alpha}^{2} d v\left(h_{\alpha}\right)
\end{aligned}
$$

Or $u_{\alpha}=\left(\frac{\lambda_{\alpha}}{N(N-2)}\right)^{(N-2) / 4} \Phi_{\alpha}$, et puisque $\tilde{v} \not \equiv 0$, on déduit facilement du lemme 4 que, quitte à extraire une sous suite, $\lim _{\alpha \rightarrow \infty} \alpha \mu_{\alpha}^{2}=0$.

(On rappelle que $\lim _{\alpha \rightarrow \infty} h_{\alpha}=\xi$ dans $C^{1}(K)$ pour $K \Subset \mathbb{R}^{N}$.)

Avec le lemme 10 et (29), (30), on obtient alors $\tilde{v} \in C^{\infty}\left(\mathbb{R}^{N}\right)$ et

$$
\Delta_{\xi} \tilde{v}=N(N-2) \tilde{v}^{p} \operatorname{sur} \mathbb{R}^{N} .
$$

Par suite, avec les résultats de [8] (voir aussi [20]), on obtient

$$
\tilde{v}(x)=\left(\frac{1}{1+|x|^{2}}\right)^{(N-2) / 2} \text {, où }|x|=d(0, x) .
$$

LEMME 11. $-\lim _{\alpha \rightarrow \infty} \int_{\omega_{\alpha}}\left|\nabla_{\xi} \tilde{u}_{\alpha}\right|^{2} d v(\xi)=\frac{N(N-2) \omega_{N}}{2^{N}}$.

Démonstration. - Avec le lemme 6, $\lim _{\alpha \rightarrow \infty} u_{\alpha}=0$ dans $C_{\text {loc }}^{1}\left(\bar{B} \backslash\left\{x_{0}\right\}\right)$. Par suite, pour tout $\delta>0, \delta \ll 1$,

$$
\lim _{\alpha \rightarrow \infty} \int_{\omega_{\alpha}}\left|\nabla_{\xi} \tilde{u}_{\alpha}\right|^{2} d v(\xi)=\lim _{\alpha \rightarrow \infty} \int_{B(0, \delta)}\left|\nabla_{\xi} \tilde{u}_{\alpha}\right|^{2} d v(\xi) .
$$

On remarque maintenant que :

$$
\forall \varepsilon>0, \exists \delta>0, \exists \alpha_{0} \gg 1 / \forall \alpha \geq \alpha_{0},\left\|g_{\alpha}-\xi\right\|_{C^{0}(B(0, \delta))}<\varepsilon .
$$

Pour le voir, il suffit d'écrire que

$$
\begin{aligned}
\left\|g_{\alpha}-\xi\right\|_{C^{0}(B(0, \delta)) \leq} & \left\|g_{\alpha}-\left(\exp _{x_{0}}\right)^{*} g\right\|_{C^{0}(B(0, \delta))} \\
& +\left\|\left(\exp _{x_{0}}\right)^{*} g-\xi\right\|_{C^{0}(B(0, \delta))} .
\end{aligned}
$$

D'après (22) on a alors $\lim _{\alpha \rightarrow \infty}\left\|g_{\alpha}-\left(\exp _{x_{0}}\right)^{*} g\right\|_{C^{0}(B(0, \delta))}=0$, et puisque $\left(\exp _{x_{0}}\right)^{*} g$ est normale en 0 , pour $\delta$ suffisamment petit on aurra $\left\|\left(\exp _{x_{0}}\right)^{*} g-\xi\right\|_{C^{0}(B(0, \delta))}<\varepsilon / 2$. 
Par suite, $\forall \varepsilon>0, \exists \delta>0, \delta \ll 1 /$

$$
\begin{aligned}
& (1-\varepsilon) \int_{B(0, \delta)}\left|\nabla_{g_{\alpha}} \tilde{u}_{\alpha}\right|^{2} d v\left(g_{\alpha}\right) \\
& \leq \int_{B(0, \delta)}\left|\nabla_{\xi} \tilde{u}_{\alpha}\right|^{2} d v(\xi) \\
& \leq(1+\varepsilon) \int_{B(0, \delta)}\left|\nabla_{g_{\alpha}} \tilde{u}_{\alpha}\right|^{2} d v\left(g_{\alpha}\right) .
\end{aligned}
$$

Reste maintenant à remarquer que pour tout $\delta>0$,

$$
\begin{aligned}
\lim _{\alpha \rightarrow \infty} \int_{B(0, \delta)}\left|\nabla_{g_{\alpha}} \tilde{u}_{\alpha}\right|^{2} d v\left(g_{\alpha}\right) & =\lim _{\alpha \rightarrow \infty} \int_{\omega_{\alpha}}\left|\nabla_{g_{\alpha}} \tilde{u}_{\alpha}\right|^{2} d v\left(g_{\alpha}\right) \\
& =\frac{N(N-2) \omega_{N}}{2^{N}} \text { d'après (27). }
\end{aligned}
$$

Le lemme est donc bien démontré.

Pour finir, on démontre le résultat suivant.

LEMME 12. $-\lim _{\alpha \rightarrow \infty} \tilde{v}_{\alpha}=\tilde{v}$ dans $L^{p+1}\left(\mathbb{R}^{N}\right)$, $\tilde{v}_{\alpha}$ étant prolongée par 0 en dehors de $\Omega_{\alpha}^{\alpha \rightarrow \infty}$.

Démonstration. - On démontre en fait un peu plus en montrant que $\lim _{\alpha \rightarrow \infty} \int_{\mathbb{R}^{N}}\left|\nabla_{\xi}\left(\tilde{v}_{\alpha}-\tilde{v}\right)\right|^{2} d v(\xi)=0$. Le résultat annoncé découle alors du théorème d'inclusion de Sobolev.

On a :

$$
\begin{aligned}
\int_{\mathbb{R}^{N}}\left|\nabla_{\xi}\left(\tilde{v}_{\alpha}-\tilde{v}\right)\right|^{2} d v(\xi)= & \int_{\Omega_{\alpha}}\left|\nabla_{\xi} \tilde{v}_{\alpha}\right|^{2} d v(\xi)+\int_{\mathbb{R}^{N}}\left|\nabla_{\xi} \tilde{v}\right|^{2} d v(\xi) \\
& -2 \int_{\Omega_{\alpha}}\left(\nabla_{\xi} \tilde{v}_{\alpha} \nabla_{\xi} \tilde{v}\right) d v(\xi)
\end{aligned}
$$

Or :

$$
\begin{aligned}
\int_{\Omega_{\alpha}}\left(\nabla_{\xi} \tilde{v}_{\alpha} \nabla_{\xi} \tilde{v}\right) d v(\xi) & =\int_{\Omega_{\alpha}} \tilde{v}_{\alpha} \Delta_{\xi} \tilde{v} d v(\xi) \\
& =N(N-2) \int_{\Omega_{\alpha}} \tilde{v}_{\alpha} \tilde{v}^{p} d v(\xi)
\end{aligned}
$$

et puisque $0 \leq \tilde{v}_{\alpha} \leq 1$ (voir (28)),

$$
\lim _{\alpha \rightarrow \infty} \int_{\Omega_{\alpha}} \tilde{v}_{\alpha} \tilde{v}^{p} d v(\xi)=\int_{\mathbb{R}^{N}} \tilde{v}^{p+1} d v(\xi) .
$$


Par ailleurs,

$$
\lim _{\alpha \rightarrow \infty} \int_{\Omega_{\alpha}}\left|\nabla_{\xi} \tilde{v}_{\alpha}\right|^{2} d v(\xi)=\lim _{\alpha \rightarrow \infty} \int_{\omega_{\alpha}}\left|\nabla_{\xi} \tilde{u}_{\alpha}\right|^{2} d v(\xi)=\frac{N(N-2) \omega_{N}}{2^{N}}
$$

d'après le lemme 11. Enfin,

$$
\int_{\mathbb{R}^{N}}\left|\nabla_{\xi} \tilde{v}\right|^{2} d v(\xi)=N(N-2) \int_{\mathbb{R}^{N}} \tilde{v}^{p+1} d v(\xi)=\frac{N(N-2) \omega_{N}}{2^{N}} .
$$

Par conséquent,

$$
\begin{aligned}
& \lim _{\alpha \rightarrow \infty} \int_{\mathbb{R}^{N}}\left|\nabla_{\xi}\left(\tilde{v}_{\alpha}-\tilde{v}\right)\right|^{2} d v(\xi) \\
& =\frac{N(N-2) \omega_{N}}{2^{N}}+\frac{N(N-2) \omega_{N}}{2^{N}}-2 \frac{N(N-2) \omega_{N}}{2^{N}}=0 .
\end{aligned}
$$

Le lemme est donc bien démontré.

\section{UNE ESTIMÉE $C^{0}$ POUR $\tilde{v}_{\alpha}$}

Les notations sont celles du paragraphe 6. On démontre le résultat suivant.

Proposition 13. - Il existe une constante $C>0$ telle que pour tout $\alpha$ et tout $x \in \Omega_{\alpha}, \tilde{v}_{\alpha}(x) \leq C \tilde{v}(x)$.

La démonstration de cette proposition passe par plusieurs étapes. On note

$$
\begin{aligned}
\tilde{h}_{\alpha} & =\tilde{v}^{4 /(N-2)} h_{\alpha} \\
\tilde{h} & =\tilde{v}^{4 /(N-2)} \xi .
\end{aligned}
$$

Là encore, à un facteur multiplicatif 4 près, $\tilde{h}$ représente la métrique standard de la sphère après projection stéréographique. Si maintenant $\tilde{w}_{\alpha}=\tilde{v}_{\alpha} / \tilde{v}$, on vérifie sans difficulté que

$$
\Delta_{\tilde{h}_{\alpha}} \tilde{w}_{\alpha}+\left(\frac{\Delta_{\tilde{h}_{\alpha}} \tilde{v}}{\tilde{v}^{p}}+\frac{\alpha \mu_{\alpha}^{2}}{\tilde{v}^{p-1}}\right) \tilde{w}_{\alpha}=N(N-2) \tilde{w}_{\alpha}^{p} \quad \text { sur } \Omega_{\alpha} .
$$

En effet, d'après (29).

$$
\frac{4(N-1)}{N-2} \Delta_{h_{\alpha}} \tilde{v}_{\alpha}+\frac{4(N-1)}{N-2} \alpha \mu_{\alpha}^{2} \tilde{v}_{\alpha}=4 N(N-1) \tilde{v}_{\alpha}^{p}
$$


et si $S\left(h_{\alpha}\right)$ désigne la courbure scalaire de $h_{\alpha}$, on peut écrire

$$
\begin{aligned}
& \frac{4(N-1)}{N-2} \Delta_{h_{\alpha}} \tilde{v}_{\alpha}+S\left(h_{\alpha}\right) \tilde{v}_{\alpha}+\left(\frac{4(N-1)}{N-2} \alpha \mu_{\alpha}^{2}-S\left(h_{\alpha}\right)\right) \tilde{v}_{\alpha} \\
& =4 N(N-1) \tilde{v}_{\alpha}^{p} .
\end{aligned}
$$

Reste alors à remarquer que

$$
\begin{aligned}
& \frac{4(N-1)}{N-2} \Delta_{h_{\alpha}} \tilde{v}_{\alpha}+S\left(h_{\alpha}\right) \tilde{v}_{\alpha}=\tilde{v}^{p}\left(\frac{4(N-1)}{N-2} \Delta_{\tilde{h}_{\alpha}} \tilde{w}_{\alpha}+S\left(\tilde{h}_{\alpha}\right) \tilde{w}_{\alpha}\right) \\
& \frac{4(N-1)}{N-2} \Delta_{h_{\alpha}} \tilde{v}+S\left(h_{\alpha}\right) \tilde{v}=S\left(\tilde{h}_{\alpha}\right) \tilde{v}^{p} .
\end{aligned}
$$

On démontre maintenant le résultat suivant.

LEMME 14. - Pour $\alpha \gg 1$ et pour tout $x \in \Omega_{\alpha}, \frac{\Delta_{h_{\alpha}} \tilde{v}}{\tilde{v}^{p}}+\frac{\alpha \mu_{\alpha}^{2}}{\tilde{v}^{p-1}} \geq 0$.

Démonstration. - Soit $\phi_{\alpha}(x)=\mu_{\alpha} x . \phi_{\alpha}$ réalise une isométrie de $\left(\Omega_{\alpha}, \mu_{\alpha}^{2} h_{\alpha}\right)$ sur $\left(\omega_{\alpha}, g_{\alpha}\right)$. Par suite, $\Delta_{h_{\alpha}} \tilde{v}=\mu_{\alpha}^{2}\left(\Delta_{g_{\alpha}}\left(\tilde{v} \circ \phi_{\alpha}^{-1}\right)\right) \circ \phi_{\alpha}$. Indépendamment, puisque les $g_{\alpha}$ sont géodésiquement normales en 0 , pour tout $u=u(r)$

$$
\Delta_{g_{\alpha}} u=\Delta_{\xi} u+u^{\prime} \partial_{r} \log \sqrt{\left|g_{\alpha}\right|}
$$

où $r=|x|$ et $\left|g_{\alpha}\right|$ est comme dans [2], chapitre 4.

De plus, voir [2] chapitre 4 et théorème $1.53,\left(\omega_{\alpha}, g_{\alpha}\right)$ et $(B, g)$ étant isométriques, il existe une constante $A>0$, indépendante de $\alpha$, telle que $\mid \partial_{r} \log \sqrt{\left|g_{\alpha}\right| \mid} \leq$ Ar. On obtient ainsi, $\Delta_{h_{\alpha}} \tilde{v} \geq \Delta_{\xi} \tilde{v}-A \mu_{\alpha}^{2} r\left|\tilde{v}^{\prime}\right|$. Par suite, puisque $\Delta_{\xi} \tilde{v}=N(N-2) \tilde{v}^{p}$ et puisque

on obtient

$$
\tilde{v}^{\prime}(x)=-(N-2)|x|\left(\frac{1}{1+|x|^{2}}\right)^{N / 2},
$$

$$
\begin{aligned}
\frac{\Delta_{h_{\alpha}} \tilde{v}}{\tilde{v}^{p}}+\frac{\alpha \mu_{\alpha}^{2}}{\tilde{v}^{p-1}} \geq N(N-2)+\alpha \mu_{\alpha}^{2}\left(1+|x|^{2}\right)^{2} \\
\quad-(N-2) A \mu_{\alpha}^{2}|x|^{2}\left(1+|x|^{2}\right) \\
=N(N-2)+\mu_{\alpha}^{2}\left(1+|x|^{2}\right)\left(\alpha+\alpha|x|^{2}-(N-2) A|x|^{2}\right) \\
=N(N-2)+\alpha \mu_{\alpha}^{2}\left(1+|x|^{2}\right)+(\alpha-(N-2) A) \mu_{\alpha}^{2}|x|^{2}\left(1+|x|^{2}\right) \\
\quad \geq 0 \quad \text { si } \alpha \geq(N-2) A .
\end{aligned}
$$

Le lemme 14 est donc bien démontré.

Du lemme 14 on déduit maintenant

$$
\Delta_{\tilde{h}_{\alpha}} \tilde{w}_{\alpha} \leq N(N-2) \tilde{w}_{\alpha}^{p} \operatorname{sur} \Omega_{\alpha}
$$

Vol. 13, $\mathrm{n}^{\circ} 1-1996$. 
On note alors

$$
\begin{aligned}
& \phi \mathrm{l}^{\prime} \text { inversion définie par } \phi(x)=\frac{x}{|x|^{2}} \\
& \Theta_{\alpha}=\phi\left(\Omega_{\alpha}\right) \\
& W_{\alpha}=\tilde{w}_{\alpha} \circ \phi \\
& H_{\alpha}=\phi^{*} \tilde{h}_{\alpha} .
\end{aligned}
$$

$\Theta_{\alpha}$ est du type $\mathbb{R}^{N} \backslash \theta_{\alpha}$ où les $\theta_{\alpha}$ sont des ouverts contenant 0 et tels que pour tout $\delta>0$, et tout $\alpha \gg 1, \theta_{\alpha} \subset B(0, \delta)$. De plus, puisque $\phi$ réalise une isométrie de $\left(\Omega_{\alpha}, h_{\alpha}\right)$ sur $\left(\Theta_{\alpha}, H_{\alpha}\right)$, l'inégalité (31) est conservée et on obtient

$$
\Delta_{H_{\alpha}} W_{\alpha} \leq N(N-2) W_{\alpha}^{p} \operatorname{sur} \Theta_{\alpha}
$$

Enfin,

$$
\begin{aligned}
\left(H_{\alpha}\right)(x)_{i j}= & \left(\frac{|x|^{2}}{1+|x|^{2}}\right)^{2} g_{\alpha}\left(\mu_{\alpha} \frac{x}{|x|^{2}}\right)_{k l} \\
& \times\left(\frac{\left(\delta_{i}^{k}|x|^{2}-2 x_{i} x^{k}\right)}{|x|^{4}} \frac{\left(\delta_{j}^{l}|x|^{2}-2 x_{j} x^{l}\right)}{|x|^{4}}\right),
\end{aligned}
$$

et ainsi (voir (20) et (21)) :

$$
\exists \lambda>0 / \text { pour tout } \alpha \text { et tout } x \in \Theta_{\alpha} \cap B, \frac{1}{\lambda} \xi \leq H_{\alpha} \leq \lambda \xi
$$

$$
\text { les } H_{\alpha} \text { sont uniformément bornées } C^{0} \operatorname{sur} \Theta_{\alpha} \cap B \text {. }
$$

On démontre maintenant le résultat suivant.

LEMME 15. - $\lim _{\alpha \rightarrow \infty} W_{\alpha}=1$ dans $L^{p+1}(B)$.

Démonstration. - On a

$$
\begin{aligned}
\int_{B}\left|W_{\alpha}-1\right|^{p+1} d v(\xi) & \leq C_{1} \int_{B}\left|W_{\alpha}-1\right|^{p+1} d v\left(H_{\alpha}\right) \quad\left(\mathrm{d}^{\prime} \text { après }(33)\right) \\
& =C_{1} \int_{\phi(B)}\left|\tilde{w}_{\alpha}-1\right|^{p+1} d v\left(\tilde{h}_{\alpha}\right) \\
& =C_{1} \int_{\phi(B)}\left|\tilde{v}_{\alpha}-\tilde{v}\right|^{p+1} d v\left(h_{\alpha}\right) \\
& \leq C_{2} \int_{\Omega_{\alpha}}\left|\tilde{v}_{\alpha}-\tilde{v}\right|^{p+1} d v(\xi)
\end{aligned}
$$

(avec (20), les $h_{\alpha}$ sont équivalents à la métrique euclidienne). 
Par suite, en vertu du lemme 12, $\lim _{\alpha \rightarrow \infty} \int_{B}\left|W_{\alpha}-1\right|^{p+1} d v(\xi)=0$.

On possède maintenant tous les renseignements nécessaires à la démonstration de la proposition.

FIN DE LA DÉMONSTRATION DE LA PROPOSITION 13. - On remarque déjà que démontrer la proposition équivaut à montrer l'existence d'une constante $C>0$ telle que pour tout $\alpha$ et tout $x \in \Omega_{\alpha}, \tilde{w}_{\alpha}(x) \leq C$. Or, pour tout $K \Subset \mathbb{R}^{N}, \lim _{\alpha \rightarrow \infty} \tilde{w}_{\alpha}=1$ dans $L^{\infty}(K)$. Par suite, démontrer la proposition revient à montrer l'existence d'une constante $C>0$ et d'un réel $R>0$ tels que pour tout $\alpha$ et tout $x \in \Omega_{\alpha}$ vérifiant $|x| \geq R, \tilde{w}_{\alpha}(x) \leq C$. Ce que l'on peut encore écrire, après inversion :

$$
\exists C>0, \exists \delta_{0}>0 / \forall \alpha \text { et } \forall x \in \Theta_{\alpha} \text { vérifiant }|x|<\delta_{0}, W_{\alpha}(x) \leq C .
$$

Soit alors $\eta \in C^{\infty}\left(\mathbb{R}^{N}\right)$ telle que $0 \leq \eta \leq 1, \eta=1$ sur $B(0, \delta / 2)$, $\eta=0$ sur $\mathbb{R}^{N} \backslash B(0, \delta), \delta>0$. En multipliant (32) par $\eta^{2} W_{\alpha}^{k}, k \geq 1$, on obtient (voir [14]) :

$$
\begin{aligned}
& \frac{4 k}{(k+1)^{2}} \int_{\Theta_{\alpha}}\left|\nabla_{H_{\alpha}}\left(\eta W_{\alpha}^{(k+1) / 2}\right)\right|^{2} d v\left(H_{\alpha}\right) \\
& \quad-\frac{2(k-1)}{(k+1)^{2}} \int_{\Theta_{\alpha}} \eta\left(\Delta_{H_{\alpha}} \eta\right) W_{\alpha}^{k+1} d v\left(H_{\alpha}\right) \\
& \quad-\frac{2}{(k+1)} \int_{\Theta_{\alpha}}\left|\nabla_{H_{\alpha}} \eta\right|^{2} W_{\alpha}^{(k+1)} d v\left(H_{\alpha}\right) \\
& \leq N(N-2) \int_{\Theta_{\alpha}} \eta^{2} W_{\alpha}^{k+p} d v\left(H_{\alpha}\right) .
\end{aligned}
$$

Les inégalités de Hölder pour le membre de droite de (35) et l'inégalité de Sobolev classique rappelée dans l'introduction permettent alors de montrer

$$
\begin{aligned}
& \int_{\Theta_{\alpha}}\left|\nabla_{H_{\alpha}}\left(\eta W_{\alpha}^{(k+1) / 2}\right)\right|^{2} d v\left(H_{\alpha}\right) \\
& \quad \leq C_{1} \int_{\Theta_{\alpha}}\left|\nabla_{H_{\alpha}}\left(\eta W_{\alpha}^{(k+1) / 2}\right)\right|^{2} d v\left(H_{\alpha}\right)+C_{2}
\end{aligned}
$$


où

$$
\begin{aligned}
C_{1}= & \frac{(k+1)^{2}}{4 k}(1+\varepsilon)\left(\int_{B(0, \delta)} W_{\alpha}^{p+1} d v\left(H_{\alpha}\right)\right)^{(p-1) /(p+1)} \\
C_{2} \leq & \frac{k-1}{2 k} \int_{\Theta_{\alpha}} \eta\left|\Delta_{H_{\alpha}} \eta\right| W_{\alpha}^{k+1} d v\left(H_{\alpha}\right) \\
& +\frac{k+1}{2 k} \int_{\Theta_{\alpha}}\left|\nabla_{H_{\alpha}} \eta\right|^{2} W_{\alpha}^{k+1} d v\left(H_{\alpha}\right) .
\end{aligned}
$$

Avec le lemme 15, on voit maintenant que pour tout $k$, on peut choisir $\delta>0, \delta \ll 1$, de sorte que $C_{1} \leq C<1, \forall \alpha$. Par suite, avec un raisonnement par récurrence du même type que celui effectué pour la démonstration du lemme 6 , on obtient :

$$
\forall q \in \mathbb{N}, \exists \delta>0, \delta \ll 1 / \text { pour tout } \alpha, \int_{B(0, \delta)} W_{\alpha}^{q} d v(\xi) \leq \text { Cte. }
$$

(On rappelle que les métriques $H_{\alpha}$ vérifient (33).)

On écrit maintenant (32) sous la forme

$$
D_{i}\left(H_{\alpha}^{i j} \sqrt{\left|H_{\alpha}\right|} D_{j} W_{\alpha}\right) \geq-N(N-2) \sqrt{\left|H_{\alpha}\right|} W_{\alpha}^{p} .
$$

D'après (33), (34) et (37) on peut appliquer le théorème 8.25 de [11]. Par suite, il existe une constante $C>0$ et il existe $\delta_{0}>0, \delta_{0} \ll 1$, tels que $W_{\alpha}(x) \leq C$ pour tout $x$ de $\Theta_{\alpha}$ vérifiant $|x| \leq \delta_{0}$. Comme annoncé, cette dernière inégalité termine de démontrer la proposition 13.

Remarque. - (1) Comme conséquence directe de la proposition 13, on obtient $\lim _{\alpha \rightarrow \infty} \tilde{v}_{\alpha}=\tilde{v}$ dans $L^{\infty}\left(\mathbb{R}^{N}\right)$.

(2) Le résultat de la proposition 13 est en fait strictement équivalent au résultat suivant : il existe une constante $C>0$ telle que pour tout $\alpha$ et tout $x \in \omega_{\alpha}, \tilde{u}_{\alpha}(x) \leq C\left(\frac{\mu_{\alpha}}{\mu_{\alpha}^{2}+|x|^{2}}\right)^{(N-2) / 2}$.

\section{CONCLUSION ET IDENTITÉ DE POHOZAEV}

On achève la programme annoncé au paragraphe 3 , et donc la démonstration du théorème, en montrant que l'existence des $\Phi_{\alpha}$ de la proposition 2 pour $\alpha$ grand conduit à une contradiction. L'étude est basée sur l'identité de Pohozaev et utilise fondamentalement la proposition 13.

Les notations sont celles du paragraphe 6. Dans toute la suite, les $C_{i}$ sont des constantes strictement positives qui ne dépendent pas de $\alpha$. On pose maintenant : 
$n=$ normale extérieure unitaire à $\partial \Omega_{\alpha}$ pour la métrique eucilidienne $\xi$ $\partial_{n} \tilde{v}_{\alpha}=$ dérivée normale de $\tilde{v}_{\alpha}$ au sens de la métrique euclidienne.

On obtient alors (voir [18] pour plus de détails)

$$
\begin{aligned}
& \int_{\Omega_{\alpha}}\left(x^{k} \partial_{k} \tilde{v}_{\alpha}\right) \Delta_{\xi} \tilde{v}_{\alpha} d v(\xi)+\frac{N-2}{2} \int_{\Omega_{\alpha}} \tilde{v}_{\alpha} \Delta_{\xi} \tilde{v}_{\alpha} d v(\xi) \\
& =-\frac{1}{2} \int_{\partial \Omega_{\alpha}}(x, n)\left(\partial_{n} \tilde{v}_{\alpha}\right)^{2} d \sigma(\xi),
\end{aligned}
$$

où $(\cdot, \cdot)$ désigne le produit scalaire euclidien.

Or $\Omega_{\alpha}$ est étoilé en 0 . Par suite, pour tout $x$ de $\partial \Omega_{\alpha},(x, n) \geq 0$. On obtient ainsi

$$
\int_{\Omega_{\alpha}}\left(x^{k} \partial_{k} \tilde{v}_{\alpha}\right) \Delta_{\xi} \tilde{v}_{\alpha} d v(\xi)+\frac{N-2}{2} \int_{\Omega_{\alpha}} \tilde{v}_{\alpha} \Delta_{\xi} \tilde{v}_{\alpha} d v(\xi) \leq 0
$$

Soit maintenant un réel $\eta>0$ tel que pour tout $X$ de $\mathbb{R}^{N}, h_{\alpha}^{i j} X_{i} X_{j} \geq$ $\eta|X|^{2}$. ( $\eta$ existe d'après (20)). On écrit que

$$
\eta \Delta_{\xi} \tilde{v}_{\alpha}=\Delta_{h_{\alpha}} \tilde{v}_{\alpha}+\left(h_{\alpha}^{i j}-\eta \delta^{i j}\right) \partial_{i j} \tilde{v}_{\alpha}-h_{\alpha}^{i j} \Gamma\left(h_{\alpha}\right)_{i j}^{k} \partial_{k} \tilde{v}_{\alpha},
$$

où les $\Gamma\left(h_{\alpha}\right)_{i j}^{k}=\frac{1}{2}\left(\partial_{i}\left(h_{\alpha}\right)_{m j}+\partial_{j}\left(h_{\alpha}\right)_{m i}-\partial_{m}\left(h_{\alpha}\right)_{i j}\right) h_{\alpha}^{m k}$ représentent les symbôles de Christoffel de $h_{\alpha}$.

Par suite, après avoir multiplié (38) par $\eta$, on obtient

$$
\begin{aligned}
& \int_{\Omega_{\alpha}}\left(x^{k} \partial_{k} \tilde{v}_{\alpha}\right) \Delta_{h_{\alpha}} \tilde{v}_{\alpha} d v(\xi)+\frac{N-2}{2} \int_{\Omega_{\alpha}} \tilde{v}_{\alpha} \Delta_{h_{\alpha}} \tilde{v}_{\alpha} d v(\xi) \\
& \quad+\int_{\Omega_{\alpha}}\left(x^{k} \partial_{k} \tilde{v}_{\alpha}\right)\left(h_{\alpha}^{i j}-\eta \delta^{i j}\right) \partial_{i j} \tilde{v}_{\alpha} d v(\xi) \\
& \quad-\int_{\Omega_{\alpha}}\left(x^{k} \partial_{k} \tilde{v}_{\alpha}\right)\left(h_{\alpha}^{i j} \Gamma\left(h_{\alpha}\right)_{i j}^{m}\right) \partial_{m} \tilde{v}_{\alpha} d v(\xi) \\
& \quad+\frac{N-2}{2} \int_{\Omega_{\alpha}} \tilde{v}_{\alpha}\left(h_{\alpha}^{i j}-\eta \delta^{i j}\right) \partial_{i j} \tilde{v}_{\alpha} d v(\xi) \\
& \quad-\frac{N-2}{2} \int_{\Omega_{\alpha}} \tilde{v}_{\alpha}\left(h_{\alpha}^{i j} \Gamma\left(h_{\alpha}\right)_{i j}^{m}\right) \partial_{m} \tilde{v}_{\alpha} d v(\xi) \leq 0 .
\end{aligned}
$$

Or $\tilde{v}_{\alpha}$ vérifie l'équation (29) (voir paragraphe 6), et ainsi

$$
\begin{aligned}
& \int_{\Omega_{\alpha}}\left(x^{k} \partial_{k} \tilde{v}_{\alpha}\right) \Delta_{h_{\alpha}} \tilde{v}_{\alpha} d v(\xi)+\frac{N-2}{2} \int_{\Omega_{\alpha}} \tilde{v}_{\alpha} \Delta_{h_{\alpha}} \tilde{v}_{\alpha} d v(\xi) \\
& =\int_{\Omega_{\alpha}}\left(x^{k} \partial_{k} \tilde{v}_{\alpha}\right)\left(N(N-2) \tilde{v}_{\alpha}^{p}-\left(\alpha \mu_{\alpha}^{2}\right) \tilde{v}_{\alpha}\right) d v(\xi) \\
& \quad+\frac{N-2}{2} \int_{\Omega_{\alpha}} \tilde{v}_{\alpha}\left(N(N-2) \tilde{v}_{\alpha}^{p}-\left(\alpha \mu_{\alpha}^{2}\right) \tilde{v}_{\alpha}\right) d v(\xi)
\end{aligned}
$$


Avec des intégrations par parties, $\tilde{v}_{\alpha}$ étant nulle sur $\partial \Omega_{\alpha}$, on obtient maintenant

$$
\begin{aligned}
& \int_{\Omega_{\alpha}}\left(x^{k} \partial_{k} \tilde{v}_{\alpha}\right) \tilde{v}_{\alpha}^{p} d v(\xi)=-\frac{(N-2)}{2} \int_{\Omega_{\alpha}} \tilde{v}_{\alpha}^{p+1} d v(\xi) \\
& \int_{\Omega_{\alpha}}\left(x^{k} \partial_{k} \tilde{v}_{\alpha}\right) \tilde{v}_{\alpha} d v(\xi)=-\frac{N}{2} \int_{\Omega_{\alpha}} \tilde{v}_{\alpha}^{2} d v(\xi) .
\end{aligned}
$$

Par suite,

$$
\begin{aligned}
& \int_{\Omega_{\alpha}}\left(x^{k} \partial_{k} \tilde{v}_{\alpha}\right) \Delta_{h_{\alpha}} \tilde{v}_{\alpha} d v(\xi)+\frac{N-2}{2} \int_{\Omega_{\alpha}} \tilde{v}_{\alpha} \Delta_{h_{\alpha}} \tilde{v}_{\alpha} d v(\xi) \\
& \quad=\alpha \mu_{\alpha}^{2} \int_{\Omega_{\alpha}} \tilde{v}_{\alpha}^{2} d v(\xi),
\end{aligned}
$$

et (39) devient

$$
\begin{aligned}
& \alpha \mu_{\alpha}^{2} \int_{\Omega_{\alpha}} \tilde{v}_{\alpha}^{2} d v(\xi)+\int_{\Omega_{\alpha}}\left(x^{k} \partial_{k} \tilde{v}_{\alpha}\right)\left(h_{\alpha}^{i j}-\eta \delta^{i j}\right) \partial_{i j} \tilde{v}_{\alpha} d v(\xi) \\
& -\int_{\Omega_{\alpha}}\left(x^{k} \partial_{k} \tilde{v}_{\alpha}\right)\left(h_{\alpha}^{i j} \Gamma\left(h_{\alpha}\right)_{i j}^{m}\right) \partial_{m} \tilde{v}_{\alpha} d v(\xi) \\
& +\frac{N-2}{2} \int_{\Omega_{\alpha}} \tilde{v}_{\alpha}\left(h_{\alpha}^{i j}-\eta \delta^{i j}\right) \partial_{i j} \tilde{v}_{\alpha} d v(\xi) \\
& -\frac{N-2}{2} \int_{\Omega_{\alpha}} \tilde{v}_{\alpha}\left(h_{\alpha}^{i j} \Gamma\left(h_{\alpha}\right)_{i j}^{m}\right) \partial_{m} \tilde{v}_{\alpha} d v(\xi) \leq 0 .
\end{aligned}
$$

On s'intéresse maintenant aux différents termes qui interviennent dans (40). Tout d'abord, en intégrant par parties, on obtient

$$
\begin{aligned}
\int_{\Omega_{\alpha}} & \left(x^{k} \partial_{k} \tilde{v}_{\alpha}\right)\left(h_{\alpha}^{i j}-\eta \delta^{i j}\right) \partial_{i j} \tilde{v}_{\alpha} d v(\xi) \\
= & \int_{\partial \Omega_{\alpha}}(x, n)\left(\partial_{n} \tilde{v}_{\alpha}\right)^{2}\left(\left(h_{\alpha}^{i j}-\eta \delta^{i j}\right) n_{i} n_{j}\right) d \sigma(\xi) \\
& -\int_{\Omega_{\alpha}}\left(\partial_{i} h_{\alpha}^{i j}\right) x^{k} \partial_{j} \tilde{v}_{\alpha} \partial_{k} \tilde{v}_{\alpha} d v(\xi) \\
& -\int_{\Omega_{\alpha}}\left(h_{\alpha}^{k j}-\eta \delta^{k j}\right) \partial_{k} \tilde{v}_{\alpha} \partial_{j} \tilde{v}_{\alpha} d v(\xi) \\
& -\int_{\Omega_{\alpha}}\left(h_{\alpha}^{i j}-\eta \delta^{i j}\right) x^{k} \partial_{i k} \tilde{v}_{\alpha} \partial_{j} \tilde{v}_{\alpha} d v(\xi)
\end{aligned}
$$


et

$$
\begin{aligned}
\int_{\Omega_{\alpha}} & \left(h_{\alpha}^{i j}-\eta \delta^{i j}\right) x^{k} \partial_{i k} \tilde{v}_{\alpha} \partial_{j} \tilde{v}_{\alpha} d v(\xi) \\
= & \int_{\partial \Omega_{\alpha}}(x, n)\left(\partial_{n} \tilde{v}_{\alpha}\right)^{2}\left(\left(h_{\alpha}^{i j}-\eta \delta^{i j}\right) n_{i} n_{j}\right) d \sigma(\xi) \\
& -\int_{\Omega_{\alpha}}\left(x^{k} \partial_{k} h_{\alpha}^{i j}\right) \partial_{i} \tilde{v}_{\alpha} \partial_{j} \tilde{v}_{\alpha} d v(\xi) \\
& -N \int_{\Omega_{\alpha}}\left(h_{\alpha}^{i j}-\eta \delta^{i j}\right) \partial_{i} \tilde{v}_{\alpha} \partial_{j} \tilde{v}_{\alpha} d v(\xi) \\
& -\int_{\Omega_{\alpha}}\left(h_{\alpha}^{i j}-\eta \delta^{i j}\right) x^{k} \partial_{i} \tilde{v}_{\alpha} \partial_{j k} \tilde{v}_{\alpha} d v(\xi)
\end{aligned}
$$

Par suite,

$$
\begin{aligned}
\int_{\Omega_{\alpha}} & \left(h_{\alpha}^{i j}-\eta \delta^{i j}\right) x^{k} \partial_{i k} \tilde{v}_{\alpha} \partial_{j} \tilde{v}_{\alpha} d v(\xi) \\
= & \frac{1}{2} \int_{\partial \Omega_{\alpha}}(x, n)\left(\partial_{n} \tilde{v}_{\alpha}\right)^{2}\left(\left(h_{\alpha}^{i j}-\eta \delta^{i j}\right) n_{i} n_{j}\right) d \sigma(\xi) \\
& -\frac{1}{2} \int_{\Omega_{\alpha}}\left(x^{k} \partial_{k} h_{\alpha}^{i j}\right) \partial_{i} \tilde{v}_{\alpha} \partial_{j} \tilde{v}_{\alpha} d v(\xi) \\
& -\frac{N}{2} \int_{\Omega_{\alpha}}\left(h_{\alpha}^{i j}-\eta \delta^{i j}\right) \partial_{i} \tilde{v}_{\alpha} \partial_{j} \tilde{v}_{\alpha} d v(\xi)
\end{aligned}
$$

et :

$$
\begin{aligned}
\int_{\Omega_{\alpha}} & \left(x^{k} \partial_{k} \tilde{v}_{\alpha}\right)\left(h_{\alpha}^{i j}-\eta \delta^{i j}\right) \partial_{i j} \tilde{v}_{\alpha} d v(\xi) \\
= & \frac{1}{2} \int_{\partial \Omega_{\alpha}}(x, n)\left(\partial_{n} \tilde{v}_{\alpha}\right)^{2}\left(\left(h_{\alpha}^{i j}-\eta \delta^{i j}\right) n_{i} n_{j}\right) d \sigma(\xi) \\
& -\int_{\Omega_{\alpha}}\left(\partial_{i} h_{\alpha}^{i j}\right) x^{k} \partial_{j} \tilde{v}_{\alpha} \partial_{k} \tilde{v}_{\alpha} d v(\xi) \\
& +\frac{N-2}{2} \int_{\Omega_{\alpha}}\left(h_{\alpha}^{i j}-\eta \delta^{i j}\right) \partial_{i} \tilde{v}_{\alpha} \partial_{j} \tilde{v}_{\alpha} d v(\xi) \\
& +\frac{1}{2} \int_{\Omega_{\alpha}}\left(x^{k} \partial_{k} h_{\alpha}^{i j}\right) \partial_{i} \tilde{v}_{\alpha} \partial_{j} \tilde{v}_{\alpha} d v(\xi)
\end{aligned}
$$


De même,

$$
\begin{aligned}
\int_{\Omega_{\alpha}} \tilde{v}_{\alpha}\left(h_{\alpha}^{i j}-\eta \delta^{i j}\right) \partial_{i j} \tilde{v}_{\alpha} d v(\xi)= & -\int_{\Omega_{\alpha}}\left(h_{\alpha}^{i j}-\eta \delta^{i j}\right) \partial_{i} \tilde{v}_{\alpha} \partial_{j} \tilde{v}_{\alpha} d v(\xi) \\
& -\int_{\Omega_{\alpha}}\left(\partial_{i} h_{\alpha}^{i j}\right) \tilde{v}_{\alpha} \partial_{j} \tilde{v}_{\alpha} d v(\xi)
\end{aligned}
$$

et :

$$
\int_{\Omega_{\alpha}}\left(\partial_{i} h_{\alpha}^{i j}\right) \tilde{v}_{\alpha} \partial_{j} \tilde{v}_{\alpha} d v(\xi)=-\frac{1}{2} \int_{\Omega_{\alpha}}\left(\partial_{i j} h_{\alpha}^{i j}\right) \tilde{v}_{\alpha}^{2} d v(\xi)
$$

Ainsi,

$$
\begin{aligned}
& \int_{\Omega_{\alpha}} \tilde{v}_{\alpha}\left(h_{\alpha}^{i j}-\eta \delta^{i j}\right) \partial_{i j} \tilde{v}_{\alpha} d v(\xi) \\
& =-\int_{\Omega_{\alpha}}\left(h_{\alpha}^{i j}-\eta \delta^{i j}\right) \partial_{i} \tilde{v}_{\alpha} \partial_{j} \tilde{v}_{\alpha} d v(\xi) \\
& \quad+\frac{1}{2} \int_{\Omega_{\alpha}}\left(\partial_{i j} h_{\alpha}^{i j}\right) \tilde{v}_{\alpha}^{2} d v(\xi) .
\end{aligned}
$$

\section{Enfin,}

$$
\begin{aligned}
& \int_{\Omega_{\alpha}} \tilde{v}_{\alpha}\left(h_{\alpha}^{i j} \Gamma\left(h_{\alpha}\right)_{i j}^{m}\right) \partial_{m} \tilde{v}_{\alpha} d v(\xi) \\
& =-\frac{1}{2} \int_{\Omega_{\alpha}}\left(\partial_{m}\left(h_{\alpha}^{i j} \Gamma\left(h_{\alpha}\right)_{i j}^{m}\right)\right) \tilde{v}_{\alpha}^{2} d v(\xi) .
\end{aligned}
$$

En reportant (41), (42) et (43) dans (40), et après simplification, on obtient maintenant :

$$
\begin{aligned}
& \alpha \mu_{\alpha}^{2} \int_{\Omega_{\alpha}} \tilde{v}_{\alpha}^{2} d v(\xi)+\frac{1}{2} \int_{\partial \Omega_{\alpha}}(x, n)\left(\partial_{n} \tilde{v}_{\alpha}\right)^{2}\left(\left(h_{\alpha}^{i j}-\eta \delta^{i j}\right) n_{i} n_{j}\right) d \sigma(\xi) \\
& \quad+\frac{1}{2} \int_{\Omega_{\alpha}}\left(x^{k} \partial_{k} h_{\alpha}^{i j}\right) \partial_{i} \tilde{v}_{\alpha} \partial_{j} \tilde{v}_{\alpha} d v(\xi)-\int_{\Omega_{\alpha}}\left(\partial_{i} h_{\alpha}^{i j}\right) x^{k} \partial_{j} \tilde{v}_{\alpha} \partial_{k} \tilde{v}_{\alpha} d v(\xi) \\
& \quad+\frac{N-2}{4} \int_{\partial \Omega_{\alpha}}\left(\partial_{i j} h_{\alpha}^{i j}\right) \tilde{v}_{\alpha}^{2} d v(\xi) \\
& \quad+\frac{N-2}{4} \int_{\Omega_{\alpha}}\left(\partial_{m}\left(h_{\alpha}^{i j} \Gamma\left(h_{\alpha}\right)_{i j}^{m}\right)\right) \tilde{v}_{\alpha}^{2} d v(\xi) \\
& \quad-\int_{\Omega_{\alpha}}\left(x^{k} h_{\alpha}^{i j} \Gamma\left(h_{\alpha}\right)_{i j}^{m}\right) \partial_{k} \tilde{v}_{\alpha} \partial_{m} \tilde{v}_{\alpha} d v(\xi) \leq 0 .
\end{aligned}
$$


Or, pour tout $x$ de $\partial \Omega_{\alpha},(x, n) \geq 0$ et $\left(h_{\alpha}^{i j}-\eta \delta^{i j}\right) n_{i} n_{j} \geq 0$. Par suite,

$$
\int_{\partial \Omega_{\alpha}}(x, n)\left(\partial_{n} \tilde{v}_{\alpha}\right)^{2}\left(\left(h_{\alpha}^{i j}-\eta \delta^{i j}\right) n_{i} n_{j}\right) d \sigma(\xi) \geq 0
$$

et :

$$
\begin{aligned}
& \alpha \mu_{\alpha}^{2} \int_{\Omega_{\alpha}} \tilde{v}_{\alpha}^{2} d v(\xi)+\frac{1}{2} \int_{\Omega_{\alpha}}\left(x^{k} \partial_{k} h_{\alpha}^{i j}\right) \partial_{i} \tilde{v}_{\alpha} \partial_{j} \tilde{v}_{\alpha} d v(\xi) \\
& \quad-\int_{\Omega_{\alpha}}\left(\partial_{i} h_{\alpha}^{i j}\right) x^{k} \partial_{j} \tilde{v}_{\alpha} \partial_{k} \tilde{v}_{\alpha} d v(\xi) \\
& \quad+\frac{N-2}{4} \int_{\Omega_{\alpha}}\left(\partial_{i j} h_{\alpha}^{i j}\right) \tilde{v}_{\alpha}^{2} d v(\xi) \\
& \quad+\frac{N-2}{4} \int_{\Omega_{\alpha}}\left(\partial_{m}\left(h_{\alpha}^{i j} \Gamma\left(h_{\alpha}\right)_{i j}^{m}\right)\right) \tilde{v}_{\alpha}^{2} d v(\xi) \\
& \quad-\int_{\Omega_{\alpha}}\left(x^{k} h_{\alpha}^{i j} \Gamma\left(h_{\alpha}\right)_{i j}^{m}\right) \partial_{k} \tilde{v}_{\alpha} \partial_{m} \tilde{v}_{\alpha} d v(\xi) \leq 0 .
\end{aligned}
$$

On remarque maintenant que $\partial_{i j} h_{\alpha}^{i j}(x)=\mu_{\alpha}^{2}\left(\partial_{i j} g_{\alpha}^{i j}\right)\left(\mu_{\alpha} x\right)$. Avec (20) et (21) on obtient alors :

$$
\left|\int_{\Omega_{\alpha}}\left(\partial_{i j} h_{\alpha}^{i j}\right) \tilde{v}_{\alpha}^{2} d v(\xi)\right| \leq C_{1} \mu_{\alpha}^{2} \int_{\Omega_{\alpha}} \tilde{v}_{\alpha}^{2} d v(\xi)
$$

De même, $\left|\partial_{m}\left(h_{\alpha}^{i j} \Gamma\left(h_{\alpha}\right)_{i j}^{m}\right)\right| \leq \operatorname{Cte} \mu_{\alpha}^{2}$, et ainsi

$$
\left|\int_{\Omega_{\alpha}} \partial_{m}\left(h_{\alpha}^{i j} \Gamma\left(h_{\alpha}\right)_{i j}^{m}\right) \tilde{v}_{\alpha}^{2} d v(\xi)\right| \leq C_{2} \mu_{\alpha}^{2} \int_{\Omega_{\alpha}} \tilde{v}_{\alpha}^{2} d v(\xi)
$$

Indépendemment, avec Taylor et puisque les $g_{\alpha}$ sont normales en 0 ,

$$
\begin{aligned}
\left(\partial_{k} h_{\alpha}^{i j}\right)(x) & =\mu_{\alpha}\left(\partial_{k} g_{\alpha}^{i j}\right)\left(\mu_{\alpha} x\right) \\
& \left.=\mu_{\alpha}\left(\left(\partial_{k} g_{\alpha}^{i j}\right)(0)+\mu_{\alpha}\left(\partial_{k l} g_{\alpha}^{i j}\right)\left(\mu_{\alpha} \theta x\right) x^{l}\right), \theta \in\right] 0,1[ \\
& =\mu_{\alpha}^{2}\left(\partial_{k l} g_{\alpha}^{i j}\right)\left(\mu_{\alpha} \theta x\right) x^{l} .
\end{aligned}
$$

Par suite, en utilisant l'équivalence des métriques $h_{\alpha}$ et $\xi$, des intégrations par parties, (20), (21) et l'équation (29) vérifiée par $\tilde{v}_{\alpha}$, on obtient 


$$
\begin{aligned}
\mid & \left.\int_{\Omega_{\alpha}} x^{k}\left(\partial_{k} h_{\alpha}^{i j}\right) \partial_{i} \tilde{v}_{\alpha} \partial_{j} \tilde{v}_{\alpha} d v(\xi)\left|\leq C_{3} \mu_{\alpha}^{2} \int_{\Omega_{\alpha}}\right| x\right|^{2}\left|\nabla_{\xi} \tilde{v}_{\alpha}\right|^{2} d v(\xi) \\
\leq & C_{4} \mu_{\alpha}^{2} \int_{\Omega_{\alpha}}|x|^{2}\left|\nabla_{h_{\alpha}} \tilde{v}_{\alpha}\right|^{2} d v\left(h_{\alpha}\right) \\
= & -C_{4} \mu_{\alpha}^{2} \int_{\Omega_{\alpha}}\left(\nabla_{h_{\alpha}}\left(|x|^{2} \nabla_{h_{\alpha}} \tilde{v}_{\alpha}\right)\right) \tilde{v}_{\alpha} d v\left(h_{\alpha}\right) \\
= & C_{4} \mu_{\alpha}^{2} \int_{\Omega_{\alpha}}|x|^{2}\left(\Delta_{h_{\alpha}} \tilde{v}_{\alpha}\right) \tilde{v}_{\alpha} d v\left(h_{\alpha}\right) \\
& -C_{4} \mu_{\alpha}^{2} \int_{\Omega_{\alpha}}\left(\nabla_{h_{\alpha}}|x|^{2} \nabla_{h_{\alpha}} \tilde{v}_{\alpha}\right) \tilde{v}_{\alpha} d v\left(h_{\alpha}\right) \\
= & C_{4} \mu_{\alpha}^{2} \int_{\Omega_{\alpha}}|x|^{2}\left(\Delta_{h_{\alpha}} \tilde{v}_{\alpha}\right) \tilde{v}_{\alpha} d v\left(h_{\alpha}\right) \\
& -C_{5} \mu_{\alpha}^{2} \int_{\Omega_{\alpha}}\left(\Delta_{h_{\alpha}}|x|^{2}\right) \tilde{v}_{\alpha}^{2} d v\left(h_{\alpha}\right) \\
\leq & C_{4} \mu_{\alpha}^{2} \int_{\Omega_{\alpha}}|x|^{2}\left(\Delta_{h_{\alpha}} \tilde{v}_{\alpha}\right) \tilde{v}_{\alpha} d v\left(h_{\alpha}\right)+C_{6} \mu_{\alpha}^{2} \int_{\Omega_{\alpha}} \tilde{v}_{\alpha}^{2} d v\left(h_{\alpha}\right) \\
= & C_{4} \mu_{\alpha}^{2} \int_{\Omega_{\alpha}}|x|^{2} \tilde{v}_{\alpha}\left(N(N-2) \tilde{v}_{\alpha}^{p}-\alpha \mu_{\alpha}^{2} \tilde{v}_{\alpha}\right) d v\left(h_{\alpha}\right) \\
& +C_{6} \mu_{\alpha}^{2} \int_{\Omega_{\alpha}} \tilde{v}_{\alpha}^{2} d v\left(h_{\alpha}\right) \\
\leq & C_{7} \mu_{\alpha}^{2} \int_{\Omega_{\alpha}} \tilde{v}_{\alpha}^{2} d v(\xi)+C_{8} \mu_{\alpha}^{2} \int_{\Omega_{\alpha}}|x|^{2} \tilde{v}_{\alpha}^{p+1} d v(\xi) . \\
& \\
& \\
&
\end{aligned}
$$

Par suite,

$$
\begin{aligned}
& \left|\int_{\Omega_{\alpha}} x^{k}\left(\partial_{k} h_{\alpha}^{i j}\right) \partial_{i} \tilde{v}_{\alpha} \partial_{j} \tilde{v}_{\alpha} d v(\xi)\right| \\
& \quad \leq C_{7} \mu_{\alpha}^{2} \int_{\Omega_{\alpha}} \tilde{v}_{\alpha}^{2} d v(\xi)+C_{8} \mu_{\alpha}^{2} \int_{\Omega_{\alpha}}|x|^{2} \tilde{v}_{\alpha}^{p+1} d v(\xi)
\end{aligned}
$$

et en raisonnant de la même façon, on montre que

$$
\begin{aligned}
& \left|\int_{\Omega_{\alpha}}\left(\partial_{i} h_{\alpha}^{i j}\right) x^{k} \partial_{j} \tilde{v}_{\alpha} \partial_{k} \tilde{v}_{\alpha} d v(\xi)\right| \\
& \quad \leq C_{9} \mu_{\alpha}^{2} \int_{\Omega_{\alpha}} \tilde{v}_{\alpha}^{2} d v(\xi)+C_{10} \mu_{\alpha}^{2} \int_{\Omega_{\alpha}}|x|^{2} \tilde{v}_{\alpha}^{p+1} d v(\xi) .
\end{aligned}
$$


Par ailleurs, toujours d'après Taylor et en utilisant l'expression des $\Gamma\left(h_{\alpha}\right)_{i j}^{k}$, on voit que pour tout $X$ de $\mathbb{R}^{N},\left|\left(x^{k} h_{\alpha}^{i j} \Gamma\left(h_{\alpha}\right)_{i j}^{m}\right) X_{k} X_{m}\right| \leq$ $C_{11} \mu_{\alpha}^{2}|x|^{2}|X|^{2}$. Par suite,

$$
\begin{aligned}
& \left|\int_{\Omega_{\alpha}}\left(x^{k} h_{\alpha}^{i j} \Gamma\left(h_{\alpha}\right)_{i j}^{m}\right) \partial_{k} \tilde{v}_{\alpha} \partial_{m} \tilde{v}_{\alpha} d v(\xi)\right| \\
& \leq C_{12} \mu_{\alpha}^{2} \int_{\Omega_{\alpha}}|x|^{2}\left|\nabla_{\xi} \tilde{v}_{\alpha}\right|^{2} d v(\xi)
\end{aligned}
$$

et, là encore,

$$
\begin{aligned}
& \left|\int_{\Omega_{\alpha}}\left(x^{k} h_{\alpha}^{i j} \Gamma\left(h_{\alpha}\right)_{i j}^{m}\right) \partial_{k} \tilde{v}_{\alpha} \partial_{m} \tilde{v}_{\alpha} d v(\xi)\right| \\
& \quad \leq C_{13} \mu_{\alpha}^{2} \int_{\Omega_{\alpha}} \tilde{v}_{\alpha}^{2} d v(\xi)+C_{14} \mu_{\alpha}^{2} \int_{\Omega_{\alpha}}|x|^{2} \tilde{v}_{\alpha}^{p+1} d v(\xi) .
\end{aligned}
$$

En reportant les relations (45) à (49) dans (44), et en simplifiant par $\mu_{\alpha}^{2}$, on obtient maintenant :

$$
\alpha \int_{\Omega_{\alpha}} \tilde{v}_{\alpha}^{2} d v(\xi) \leq C_{15} \int_{\Omega_{\alpha}} \tilde{v}_{\alpha}^{2} d v(\xi)+C_{16} \int_{\Omega_{\alpha}}|x|^{2} \tilde{v}_{\alpha}^{p+1} d v(\xi)
$$

Pour conclure, et donc obtenir la contradiction recherchée, il reste à remarquer que :

$$
\int_{\Omega_{\alpha}} \tilde{v}_{\alpha}^{2} d v(\xi) \geq \frac{1}{2} \int_{B} \tilde{v}^{2} d v(\xi)>0 \quad \text { pour } \alpha \gg 1
$$

(puisque les $\tilde{v}_{\alpha}$ convergent uniformément vers $\tilde{v}$ sur tout compact de $\mathbb{R}^{N}$ )

$$
\begin{gathered}
\int_{\Omega_{\alpha}}|x|^{2} \tilde{v}_{\alpha}^{p+1} d v(\xi) \leq C_{17} \int_{\mathbb{R}^{N}}|x|^{2} \tilde{v}^{p+1} d v(\xi) \\
\leq C_{18} \text { dès que } N \geq 3
\end{gathered}
$$

(d'après la proposition 13 ).

Par suite, (50), (51) et (52) imposent $\alpha \leq$ Cte. Les $\Phi_{\alpha}$ de la proposition 2 n'existent donc pas pour $\alpha$ grand, et le théorème est démontré. 


\section{APPENDICES}

\section{APPENDICE 1}

$(M, g)$ désignant une variété riemannienne compacte à bord de dimension $N \geq 3$, on note $H_{0,1}(M)$ l'espace de Sobolev complété de $\mathcal{D}(M)$, l'espace des fonctions $C^{\infty}$ à support compact dans $M \backslash \partial M$, pour la norme

$$
\|u\|^{2}=\int_{M}\left|\nabla_{g} u\right|^{2} d v(g)+\int_{M} u^{2} d v(g) .
$$

La question devient : montrer qu'il existe une constante $C>0$ telle que

$$
\begin{aligned}
& \text { pour tout } u \in H_{0,1}(M), \\
& \left(\int_{M}|u|^{p+1} d v(g)\right)^{2 /(p+1)} \\
& \quad \leq S_{N} \int_{M}\left|\nabla_{g} u\right|^{2} d v(g)+C \int_{M} u^{2} d v(g) .
\end{aligned}
$$

La réduction du problème présentée dans le paragraphe 2 reste valable pour cette question. Par suite :

THÉORÈME. - Soit $(M, g)$ une variété riemannienne compacte à bord de dimension $N \geq 3$. Il existe alors une constante $C>0$ pour laquelle (53) est vérifiée.

\section{APPENDICE 2}

On démontre une version modifiée du corollaire 8.36 de [11].

Lemme. - Pour $\alpha \gg 1$, on considère la famille $\left(D_{\alpha}\right)$ de domaines de $\mathbb{R}^{N}$ définis par l'intersection d'une boule de rayon 1 et d'une boule de rayon $\alpha$, dont un modèle peut être obtenu de la façon suivante : $D_{\alpha}=B_{1} \cap B_{\alpha}$

$$
\begin{array}{ll}
\text { où } & B_{1}=\left\{(x, y) \in \mathbb{R} \times \mathbb{R}^{N-1} / x^{2}+|y|^{2} \leq 1\right\} \\
& B_{\alpha}=\left\{(x, y) \in \mathbb{R} \times \mathbb{R}^{N-1} /\left(x+\sqrt{\alpha^{2}-1}\right)^{2}+|y|^{2} \leq \alpha^{2}\right\} .
\end{array}
$$

On note $\partial D_{\alpha}^{-}=\partial D_{\alpha} \cap B_{1}, \partial D_{\alpha}^{+}=\partial D_{\alpha} \cap B_{\alpha}$, et si $\left(x_{0}, 0\right) \in \partial D_{\alpha}^{+}$, on note $O_{\alpha}$ l'intersection de $D_{\alpha}$ avec la boule de centre $\left(x_{0}, 0\right)$ et de rayon $\frac{1}{2}$. 
Soit maintenant $\left(g_{\alpha}\right)$ une famille de métriques définies sur un voisinage ouvert de $\bar{D}_{\alpha}$ telles que

$$
\frac{1}{\lambda} \xi \leq g_{\alpha} \leq \lambda \xi(\xi \text { la métrique euclidienne })
$$

et soit $\left(v_{\alpha}\right)$ une famille de solutions régulières sur $D_{\alpha}$ des équations

$$
\begin{aligned}
& \Delta_{g_{\alpha}} v_{\alpha}+h_{\alpha} v_{\alpha}=f_{\alpha} \\
& v_{\alpha}=0 \quad \text { sur } \partial D_{\alpha}^{+}
\end{aligned}
$$

où $h_{\alpha}$ et $f_{\alpha}$ appartiennent à $C^{0}\left(D_{\alpha}\right)$.

$S i$ : (i) les métriques $g_{\alpha}$ sont uniformément bornées $C^{1}$ sur $D_{\alpha}$

(ii) les fonctions $h_{\alpha}$ et $f_{\alpha}$ sont uniformément bornées $C^{0}$ sur $D_{\alpha}$ alors il existe une constante $C$, indépendante de $\alpha$, telle que

$$
\left\|v_{\alpha}\right\|_{C^{1}\left(O_{\alpha}\right)} \leq C\left(\operatorname{Sup}_{D_{\alpha}}\left|v_{\alpha}\right|+\operatorname{Sup}_{D_{\alpha}}\left|f_{\alpha}\right|\right) \text {. }
$$

$\left(C\right.$ ne dépend en fait que de $N$, de la borne $C^{1}$ des $g_{\alpha}$, de la borne $C^{0}$ des $h_{\alpha}$ et de $\lambda$.)

Démonstration. - Pour $y \in \mathbb{R}^{N-1}$, on note $x_{\alpha}^{-}(y)$ l'unique point de $\partial D_{\alpha}^{-}$ de coordonnée $y$ sur $\mathbb{R}^{N-1}$, et on note $x_{\alpha}^{+}(y)$ l'unique point de $\partial D_{\alpha}^{+}$de coordonnée $y$ sur $\mathbb{R}^{N-1}$.

On considère maintenant la famille d'homéomorphismes $\theta_{\alpha}: D_{\alpha} \rightarrow D_{1}$ définies par

$$
\theta_{\alpha}(x, y)=\left(\frac{x_{\alpha}^{-}(y)\left(x_{\alpha}^{+}(y)-x\right)}{x_{\alpha}^{+}(y)-x_{\alpha}^{-}(y)}, y\right) .
$$

On vérifie facilement que les $\theta_{\alpha}$, restreints à

$$
D_{\alpha}^{\varepsilon}=\left\{(x, y) \in D_{\alpha} /|y|<1-\varepsilon\right\}, \varepsilon>0, \varepsilon \ll 1,
$$

sont des difféomorphismes sur leurs images, et qu'il existe $C(\varepsilon)$, indépendante de $\alpha$, telle que pour tout $(x, y) \in D_{\alpha}^{\varepsilon}$ :

$$
\frac{1}{C(\varepsilon)} \leq\left\|d \theta_{\alpha}(x, y)\right\| \leq C(\varepsilon) \text {. }
$$

Sur $D_{1}$ on considère maintenant la famille d'équations

$$
\begin{aligned}
& \Delta_{k_{\alpha}} w_{\alpha}+\left(h_{\alpha} \circ \theta_{\alpha}^{-1}\right) w_{\alpha}=f_{\alpha} \circ \theta_{\alpha}^{-1} \\
& w_{\alpha}=0 \quad \operatorname{sur} T
\end{aligned}
$$

où $k_{\alpha}=\left(\theta_{\alpha}^{-1}\right)^{*} g_{\alpha}, w_{\alpha}=v_{\alpha} \circ \theta_{\alpha}^{-1}$ et $T=\left\{(0, y) /(0, y) \in B_{1}\right\}$.

Compte tenu de (54), on se trouve dans les conditions d'application du corollaire 8.36 de [11]. Par suite,

et en utilisant (54),

$$
\left\|v_{\alpha}\right\|_{C^{1}\left(\theta_{\alpha}\left(B_{\alpha}\right)\right)} \leq C\left(\operatorname{Sup}_{D_{\alpha}}\left|v_{\alpha}\right|+\operatorname{Sup}_{D_{\alpha}}\left|f_{\alpha}\right|\right) .
$$

$$
\left\|v_{\alpha}\right\|_{C^{1}\left(O_{\alpha}\right)} \leq C\left(\operatorname{Sup}_{D_{\alpha}}\left|v_{\alpha}\right|+\operatorname{Sup}_{D_{\alpha}}\left|f_{\alpha}\right|\right) .
$$

Le lemme est donc bien démontré. 


\section{RÉFÉRENCES}

[1] R. A. Adams, Sobolev spaces, Academic press, Pure and Applied Mathematics, vol. 65, 1978.

[2] T. AubIN, Nonlinear Analysis on Manifolds, Monge-Ampère equations, Berlin, SpringerVerlag, 1982, Grudlehern Math. Wiss., vol. 252.

[3] T. Aubin, Problèmes isopérimétriques et espace de Sobolev, Journal of Differential Geometry, vol. 11, 1976, p. 573-598.

[4] T. Aubin, Équations différentielles non linéaires et problème de Yamabe concernant la courbure scalaire, Journal de Mathématiques Pures et Appliquées, vol. 55, 1976, p. 269-296.

[5] A. Besse, Einstein manifolds, Springer-Verlag, vol. 10, 1987.

[6] H. BrezIs, Elliptic equations with limiting Sobolev exponents, the impact of topology, Comm. Pure and Appl. Math., vol. XXXIX, 1986, p. 17-39.

[7] P. Cherrier, Meilleures constantes dans des inégalités relatives aux espaces de Sobolev, Bull. Sci. Math., vol. 108, 1984, p. 225-262.

[8] L. A. Caffarelli, B. Gidas et J. SPRUCK, Asymptotic symmetry and local behavior of semilinear elliptic equations with Sobolev growth, Comm. Pure and Appl. Maths., vol. XLII, 1989, p. 271-297.

[9] S. GaLlot, Inégalités isopérimétriques sur les variétés riemanniennes, Astérisques, 1988, p. $163-164$

[10] B. GidAs, W. M. Ni et L. NiRENBERG, Symmetry and related properties via the maximum principle, Communications in Mathematical Physics, vol. 68, 1979, p. 209-243.

[11] D. Gilbarg et N. S. Trudinger, Elliptic partial differential equations of second order, Berlin, Springer-Verlag, Second edition, 1983, Grundleher Math. Wiss., vol. 224.

[12] Z. C. HAN, Asymptotic approach to singular solutions for nonlinear elliptic equations involving critical Sobolev exponent, Annales de l'Institut Henri Poincaré, Analyse non linéaire, vol. 8, 1991, p. 159-174.

[13] E. Hebey, Changements de métriques conformes sur la sphère, Le problème de Nirenberg, Bull. Sc. Math., vol. 114, 1990, p. 215-242.

[14] E. HebEY, La méthode d'isométries-concentration dans le cas d'un problème non linéaire sur la variétés riemanniennes compactes à bord avec exposant critique de Sobolev, Bull. Sc. Math., vol. 116, 1992, p. 35-51.

[15] E. Hebey, Courbure scalaire et géométrie conforme, Journal of Geometry and Physics, vol. 10, 1993, p. 345-380.

[16] E. Hebey et M. Vaugon, Meilleures constantes dans le théorème d'inclusion de Sobolev et multiplicité pour les problèmes de Nirenberg et Yamabe, Indiana University Mathematics Journal, vol. 41, 1992, p. 377-407.

[17] S. KoBAYASH et K. Nomizu, Foundations of differential geometry, Interscience tracts in pure and applied mathematics, $\mathrm{n}^{\circ} 15$, John Wiley \& Sons.

[18] J. L. KAZDAN et F. W. WARNER, Remarks on some quasilinear elliptic equations, Comm. Pure Appl. Math., vol. 28, 1975, p. 567-597.

[19] P. L Lions, The concentration-compactness principle in the calculus of variations, Rev. Math. Iberoamericano, vol. 1.1, 1985, p. 145-201, et vol. 1.2, 1985, p. 45-121.

[20] M. ОвAтA, The conjectures on conformal transformations of riemannian manifolds, J. Diff. Geom., vol. 6, 1971, p. 247-258.

[21] M. STRUwE, A global compactness result for elliptic boundary value problems involving limiting nonlinearities, Math. Z., vol. 187, 1984, p. 511-517.

[22] P. SACKS et K. UhlenbeCK, On the existence of minimal immersions of 2-sphères, Ann. of Math., vol. 113, 1981, p. 1-24.

[23] G. Talenti, Best constant in Sobolev inequality, Ann. Math. Pure Appl., vol. 110, 1976, p. 353-372.

[24] M. VAugon, Transformation conforme de la courbure scalaire sur une variété riemannienne compacte, Journal of Functional Analysis, vol. 71, 1987, p. 182-194. 
[25] H. C. WENTE, Large solutions to the volume constrained Plateau problem, Arch. Rat. Mech. Anal., vol. 75, 1980 , p. 59-77.

(Manuscrit reçu le 22 mars 1994;

révisé le 2 juin 1994.)

Vol. 13, n $1-1996$. 